\title{
Dynamic rupture propagation on fault planes with explicit representation of short branches
}

\author{
Xiao Ma, Ahmed Elbanna* \\ Department of Civil and Environmental Engineering, University of Illinois, Urbana-Champaign, IL, USA
}

\section{A R T I C L E I N F O}

\section{Article history:}

Received 6 February 2019

Received in revised form 27 June 2019

Accepted 3 July 2019

Available online $\mathrm{xxxx}$

Editor: M. Ishii

\section{Keywords:}

earthquake mechanics

dynamic rupture

secondary faults

high frequency generation

\begin{abstract}
A B S T R A C T
An active fault zone is home to a plethora of complex structural and geometric features that are expected to affect earthquake rupture nucleation, propagation, and arrest, as well as interseismic deformation. Simulations of these complexities have been largely done using continuum plasticity or scalar damage theories. In this paper, we use a highly efficient novel hybrid finite element-spectral boundary integral equation scheme to investigate the dynamics of fault zones with small scale pre-existing branches as a first step towards explicit representation of anisotropic damage features in fault zones. The hybrid computational scheme enables exact near-field truncation of the elastodynamic field allowing us to use high resolution finite element discretization in a narrow region surrounding the fault zone that encompasses the small scale branches while remaining computationally efficient. Our results suggest that the small scale branches may influence the rupture in ways that may not be realizable in homogenized continuum models. Specifically, we show that these short secondary branches significantly affect the post event stress state on the main fault leading to strong heterogeneities in both normal and shear stresses and also contribute to the enhanced generation of high frequency radiation. The secondary branches also affect off-fault plastic strain distribution and suggest that co-seismic inelasticity is sensitive to pre-existing damage features. We discuss our results in the larger context of the need for modeling earthquake ruptures with high resolution fault zone physics.
\end{abstract}

(C) 2019 Elsevier B.V. All rights reserved.

\section{Introduction}

The internal structure of fault zones in the upper continental crust exhibits considerable complexity. Mature faults consist of several basic structural elements including: (i) A zone of concentrated shear, the fault core, which is often defined by the presence of extremely comminuted gouge; (ii) A damage zone, with the primary fault core centralized in or bordering that damage zone, in addition to a segmented network of several secondary cores within the damage zone. Damage zones display a greater intensity of deformation relative to the surrounding host rock, and contain features such as secondary faults and fractures, microfractures, folded strata, and comminuted grains; and (iii) host country rock with little or no damage. In general, the intensity of damage increases towards the fault core and the transition from undeformed host rock to damage zone rock is often gradual Ben-Zion and Sammis

\footnotetext{
* Corresponding author.

E-mail addresses: xiaoma5@illinois.edu (X. Ma), elbanna2@illinois.edu (A. Elbanna).
}

(2003); Chester et al. (1993); Savage and Brodsky (2011). Overall, fault zones exhibit a combination of distributed damage as well as discrete anisotropic secondary fractures of different orientations and density Rowe et al. (2018).

Off-fault damage has been investigated extensively using numerical models that implement either off-fault plastic strain accumulation Andrews (2005); Dunham et al. (2011a,b); Hok et al. (2010); Templeton and Rice (2008), or continuum damage evolution Ben-Zion and Shi (2005); Bhat et al. (2012); Xu et al. (2015a). The starting point in both approaches is a virgin material that has not experienced damage before. Furthermore, both approaches are found to be prone to numerical localization and have been, for the large part, constrained to scalar damage variables or isotropic formulations Duru and Dunham (2016); Uphoff et al. (2017). Except for a few pioneering studies, for example Dunham et al. (2011b); Shi and Day (2013); Tal et al. (2018), that considered off-fault dissipation generated by rough fault surfaces, most of the prior studies considered planar faults with no structural complexity. In particular, the effect of pre-existing anisotropic damage features on rupture dynamics, in both the elastic and inelastic regimes, remains an area that is under-studied. 
An exception to the aforementioned discussion has been the investigation of the critical problem of the influence of a fault branch on the termination or continued propagation of rupture on the main fault Bhat et al. (2004); Biegel et al. (2007); Kame et al. (2003); Poliakov et al. (2002); Rousseau and Rosakis (2009); Suzuki (2013). These studies suggest that the rupture may continue to propagate on the main fault without jumping to the branch, or propagate on both the main and secondary faults, or terminate on the main fault and continue on the branch. The fate of the rupture depends on the angle of the branch, the background stress field, and the rupture propagation speed. However, to the best of our knowledge, all these studies have been limited to a single long branch. Short and repeated branches that are routinely mapped in fault zones Rempe et al. (2013); Rowe et al. (2018) are largely neglected or homogenized as an effective damage variable. An outstanding challenge in explicit modeling of these anisotropic secondary features has been largely attributed to the prohibitive computational cost in terms of problem size, runtime, and memory requirements of domain-based methods such as finite element or finite difference techniques.

Domain based modeling approaches are very versatile in handing complex geometries and material nonlinearities compared to boundary-based methods such as the spectral boundary integral equation. However, to capture small scale details associated with short fault branches, a very fine mesh must be used to resolve the complex boundaries as well as the multiple stress concentration regions associated with the propagating rupture tips. This fine mesh is generally carried out for a significant portion of the domain to appropriately propagate the seismic waves and avoid artificial reflection from varying the mesh size over small distances. Furthermore, the simulation domain has to be truncated at some distance by imposing absorbing boundary conditions Berenger (1994); Bettess (1977); Lysmer and Kuhlemeyer (1969) far enough from the fault so that reflections from these boundaries do not affect the solution on the fault plane during the simulation time of interest. As a result, the computation cost of a domainbased method grows as $(L / d x)^{3}$ in 2D and $(L / d x)^{4}$ in 3D, making it very challenging to incorporate small scale physics in large scale simulations.

A novel approach in addressing the above challenge has been recently presented by Klinger et al. (2018), who used discrete finite element model to study co-seismic off-fault damage generation resolving complex rupture process. The numerical method presented by Klinger et al. enabled generation of co-seismic damage patterns that localize into a set of nearly periodic parallel branches. While their formulation is based on continuum damage theory, the damage parameter may numerically localize and eventually be replaced by a slip-weakening crack. Earlier work by Ando and Yamashita (2007) has also provided a framework for spontaneous generation of off-plane faults using a novel formulation of the boundary integral method. However, what continues to be missing in this work is the effect of pre-existing secondary cracks, which is expected to influence the dynamic rupture characteristics high frequency radiation and new damage generation, in a way that is different from co-seismically generated damage in a virgin material. In this paper we plan to address this missing piece using a novel numerical scheme that enables incorporating high resolution fault zone physics and geometric structures in dynamic rupture calculations.

Here, we use our recently developed hybrid computational scheme that combines a domain-based numerical method which is used to discretize a confined region encompassing the fault plane and all its related structural and material complexities, with an independent spectral boundary integral formulation that models the exterior linear elastic half spaces Hajarolasvadi and Elbanna (2017); Ma et al. (2018). This approach overcomes the limitations of the domain-based methods by limiting the discretization to only a subset of the whole domain but benefits from their flexibility in modeling complex geometry and material nonlinearity. The reduction in the size of the domain to be discretized enables us to use higher resolution within the fault zone to resolve the complexity of the secondary branches while saving computational cost and not compromising the accuracy of long range elastodynamic interactions, which are handled exactly using the spectral boundary integrals. In our prior work Ma et al. (2018) we have discussed the novelty of our hybrid formulation in the context of existing literature on coupling boundary and bulk numerical schemes as in Bielak et al. (2003); Yoshimura et al. (2003). In this paper, we will use the hybrid scheme to investigate the dynamics of rupture propagation on a fault plane with multiple short branches mimicking the fish bone architecture idealized in Poliakov et al. (2002); Sowers et al. (1911).

\section{Numerical method and model setup}

\subsection{Hybrid finite element-spectral integral equation method}

We solve the initial boundary value problem of dynamic fracture using the recently developed hybrid method Ma et al. (2018). The hybrid method is a combination of the FEM (finite element method) and SBI (spectral boundary integral method), although any other domain-based method may be used in lieu of FEM. In the hybrid method, all nonlinearities, such as fault surface roughness or material nonlinearity, as well as small-scale heterogeneities, are contained in a virtual strip of a certain width that is introduced for computational purposes only (Fig. 1(a)). Appropriate meshing techniques are then used to discretize and model this strip using FEM. The step-by-step time integration approach for the fault nodes is a central-difference explicit formulation as follows:

$$
\begin{aligned}
\dot{u}^{n+1 / 2} & =\dot{u}^{n-1 / 2}+\Delta t M^{-1}\left(T^{n}-f^{n}\right) \\
u^{n+1} & =u^{n}+\Delta t \dot{u}^{n+1 / 2}
\end{aligned}
$$

where represents the partial derivative with respect to time and the superscript $n$ indicates the time step index. $M$ is the lumped mass matrix. $T_{n}$ is the traction on the fault interface based on the fault discontinuity condition. The fault discontinuity condition is implemented using the Traction at Split Nodes (TSN) method Day (1982). $f$ is the internal force due to the deformation of the solid and $\Delta t$ the time step.

For the interior nodes in the FEM domain, the step-by-step time integration approach is as follows:

$$
\begin{aligned}
\dot{u}^{n+1 / 2} & =\dot{u}^{n-1 / 2}+\Delta t M^{-1}\left(-f^{n}\right) \\
u^{n+1} & =u^{n}+\Delta t \dot{u}^{n+1 / 2}
\end{aligned}
$$

The rest of the domain, which is homogeneous and linear-elastic, may be modeled as two half spaces coupled with this strip on each side $\left(S^{+}, S^{-}\right)$. The elastodynamic response of these half spaces is modeled using the SBI technique. Throughout the simulation, the two methods communicate along the virtual boundaries of the strip by exchanging displacement and traction boundary conditions. The spectral formulation for this method gives an exact form of such a relationship in the Fourier domain. We use the spectral formulation introduced in Geubelle and Rice (1995), where the elastodynamic analysis of each half space is carried out separately. In view of the hybrid method, where SBI constitutes a boundary condition to the FEM model, we focus the description on modeling a half-space. The relationship between the traction $\tau_{i}$ and the 


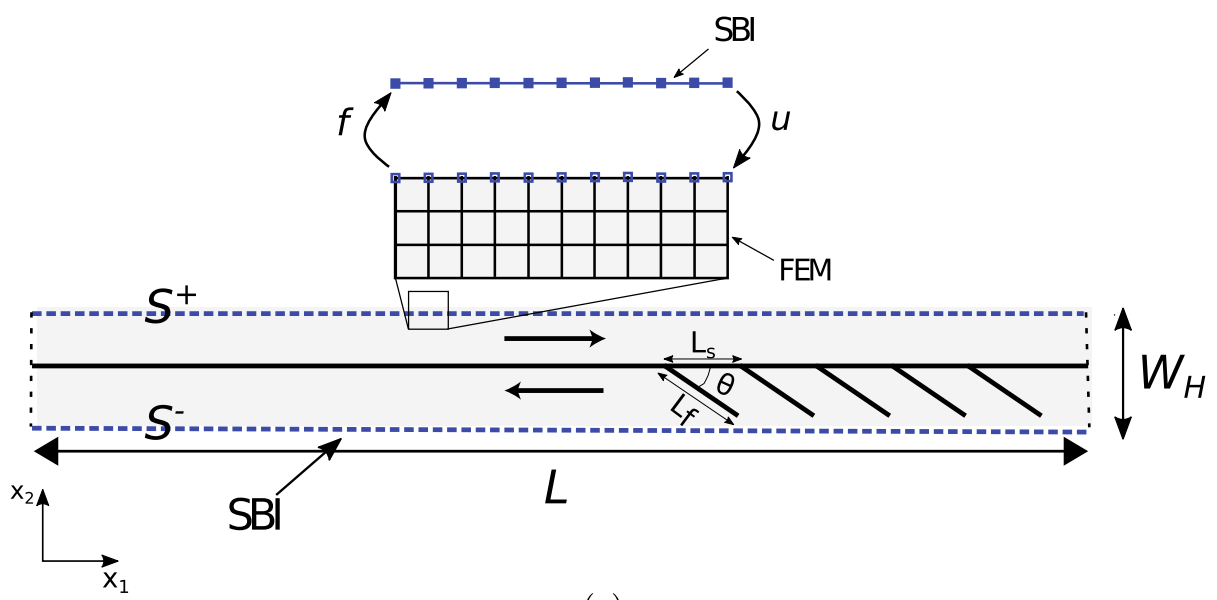

(a)

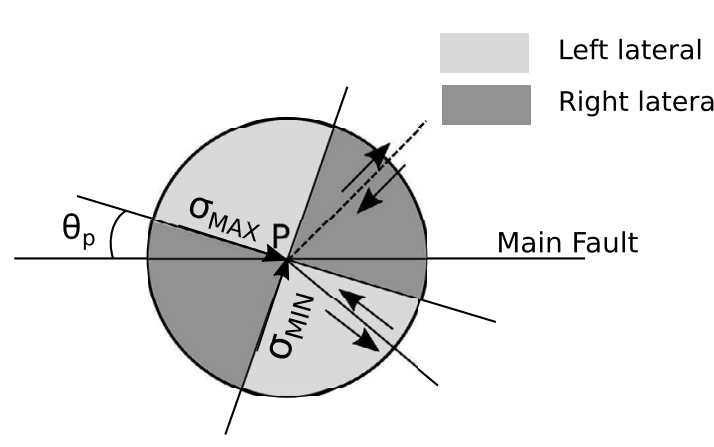

(b)

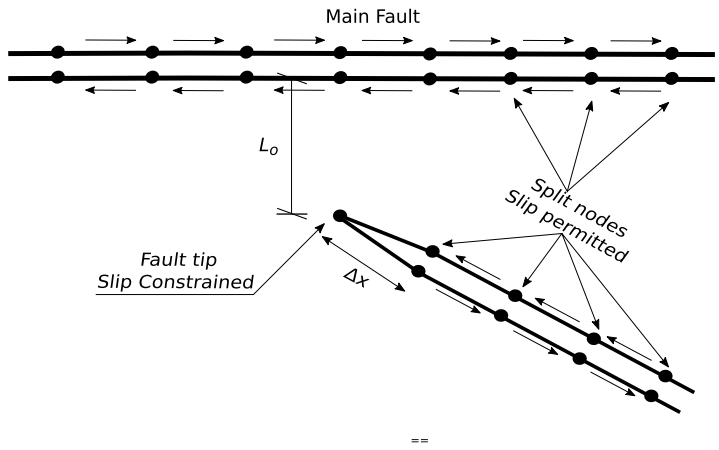

(c)

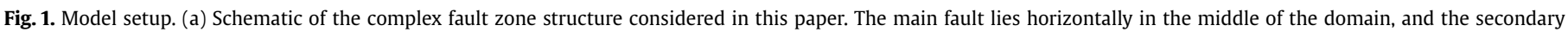

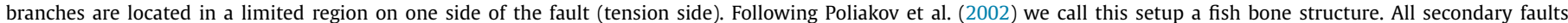

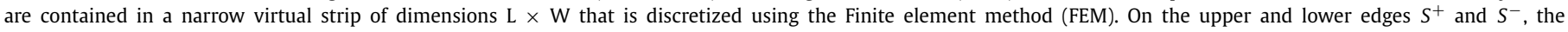

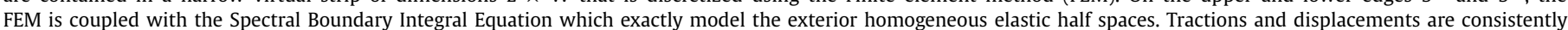

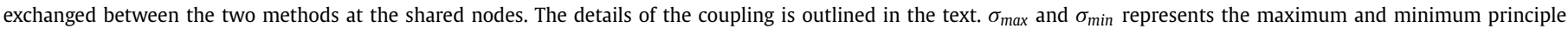

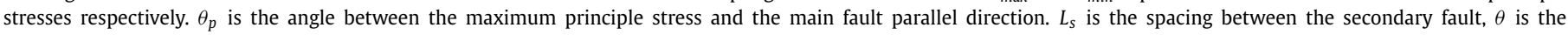

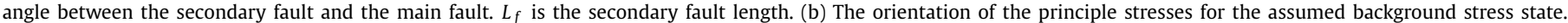

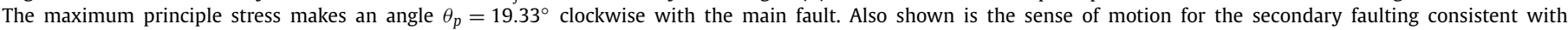

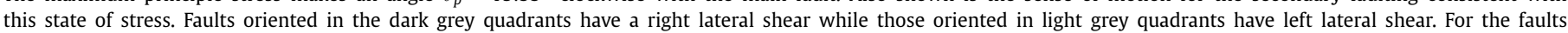

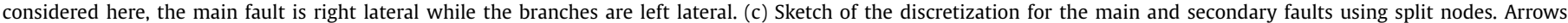
represent the sense of shear. The secondary fault is shifted $L_{o}$ away from the main fault. The slip is constrained to be zero at the tips of the secondary fault.

resulting displacements at the boundary of a half-space may be expressed as

$$
\begin{aligned}
& \tau_{1}^{ \pm}\left(x_{1}, t\right)=\tau_{1}^{0 \pm}\left(x_{1}, t\right) \mp \frac{\mu}{c_{s}} \dot{u}_{1}^{ \pm}\left(x_{1}, t\right)+f_{1}^{ \pm}\left(x_{1}, t\right) \\
& \tau_{2}^{ \pm}\left(x_{1}, t\right)=\tau_{2}^{0 \pm}\left(x_{1}, t\right) \mp \frac{(\lambda+2 \mu)}{c_{p}} \dot{u}_{2}^{ \pm}\left(x_{1}, t\right)+f_{2}^{ \pm}\left(x_{1}, t\right)
\end{aligned}
$$

where subscripts 1 and 2 represent fault-parallel and fault-normal direction respectively, \pm represents upper and lower half-plane, $c_{p}$ is the pressure wave speed, $c_{s}$ is the shear wave speed, $\tau_{i}^{0}$ indicates the externally applied load (i.e., at infinity); and $f_{i}$ are linear functionals of the prior deformation history and are computed by the time convolution in the Fourier domain.

The coupling of the two methods is done as follows. The FEM and SBI share nodes at the virtual boundaries introduced to truncate the FEM domain. While FEM provides SBI with the tractions along the virtual boundary, SBI returns the displacement that is to be imposed on $S^{ \pm}$of FEM. The detailed step-by-step procedure is as follows

1. Solve full time step within the FEM by solving Eq. (1)-(2) (FEM interior nodes only).

2. Set interface tractions in the SBI equal to the internal force from FEM: $\tau_{i}^{n, \mathrm{SBI}}=f_{i}^{n, \mathrm{FEM}}$, where $f_{i}^{n}$ is given through Eq. (1).
3. Solve full time step within SBI by solving Eq. (5) for velocity and apply explicit integration scheme to get displacements.

4. Set displacements of the shared nodes in FEM equal to displacement in SBI: $u_{i}^{n+1, \mathrm{FEM}}=u_{i}^{n+1, \mathrm{SBI}}$.

5. Return to Step 1 to advance to the next time step.

For a full description of the hybrid scheme, its verification, and some of its prior applications please refer to Ma et al. (2018).

\subsection{Model setup}

\subsubsection{Material and friction model}

In this paper, we consider both linear elastic material and elasto-plastic material.

\section{Linear elastic material}

A 2D plane strain elastic model is used to describe the elastic material behavior. The constitutive equation for the linear elastic material is as follows:

$\sigma_{i j}=\lambda \delta_{i j} \varepsilon_{k k}+2 \mu \varepsilon_{i j}$

where $\varepsilon_{i j}$ is the infinitesimal strain tensor and $\mu, \lambda$ are the Lamé parameters. 


\section{Elasto-plastic material}

In this paper, we also consider the off-fault material to be idealized with the Drucker-Prager plasticity model Drucker and Prager (1952). The Drucker-Prager model is closely related to the MohrCoulomb model. It describes inelastic deformation in brittle solids arising from frictional sliding of microcracks Rudnicki and Rice (1975); Templeton and Rice (2008). We use the Drucker-Prager plasticity model to mimic the inelastic effects on dynamic rupture from cracks on scales that are smaller than the scale of branches. The yield function of the Drucker-Prager plasticity model is given by Eq. (7),

$F\left(\sigma_{i j}\right)=\sqrt{J_{2}}-\left(A+B I_{1}\right)$

Here, $I_{1}=\sigma_{k k}$ is the first invariant of the Cauchy stress $\sigma_{i j}$ and $J_{2}=s_{i j} s_{i j} / 2$ is the second invariant of the deviatoric stress tensor $s_{i j}=\sigma_{i j}-\left(\sigma_{k k} / 3\right) \delta_{i j}$. Following Templeton and Rice (2008), we take the intermediate principal stress, in the Drucker-Prager formulation, to be the average of the maximum and the minimum principle stress. The constants $A$ and $B$ are determined from experiments and are functions of the cohesion $c$ and the angle of internal friction $\phi$ that are used to describe the Mohr-Coulomb yield surface. When $F\left(\sigma_{i j}\right)<0$, the material response is elastic.

Plastic flow is partitioned between various components of the plastic strain rate tensor by the flow rule. Neglecting the effect of plastic dilatancy we have:

$\dot{\epsilon}_{i j}^{p}=\dot{\epsilon}_{p}^{e q} s_{i j} /\left(2 \sqrt{J_{2}}\right)$

Where $\dot{\epsilon}_{p}^{e q}=\sqrt{2 \dot{\epsilon}_{i j}^{p} \dot{\epsilon}_{i j}^{p}}$ is the equivalent plastic strain rate. The equivalent plastic strain $\epsilon_{p}^{e q}$ is defined through $\dot{\epsilon}_{p}^{e q}=d \epsilon_{p}^{e q} / d t$.

\section{Slip-weakening friction model}

In this paper, all the faults are governed by the slip-weakening friction law Ida (1972). The frictional strength is given by

$f(D)= \begin{cases}f_{s}-\left(f_{s}-f_{d}\right) D / D_{c}, & D<D_{c} \\ f_{d}, & D \geq D_{c}\end{cases}$

where $f_{s}$ and $f_{d}$ are the static and dynamic frictional coefficients and $D_{c}$ the critical slip required for stress to reach the dynamic value. Continuity of displacements at the fault is enforced (i.e., no slip) if the shear traction is lower than frictional strength, otherwise local slip occurs. Uenishi and Rice (2003) defined the characteristic length scale for frictional instability on linear slipweakening faults. We base our reference length scale for normalizing the spatial scales in our problem on this characteristic length scale term as shown in Eq. (10) (omitting the constant term from Uenishi and Rice (2003)).

$L_{c}=\frac{\mu D_{c}}{\tau_{s}-\tau_{d}}$

Here, $\mu$ is the shear modulus, $D_{c}$ is the characteristic slip distance, $\tau_{s}$ is the static frictional stress and $\tau_{d}$ is the dynamic frictional stress.

\section{Normal stress regularization}

Due to the complex topology of our fault network, normal stress may be altered on the main fault as well as secondary faults. In order to avoid numerical instability and for the friction model to be compatible with laboratory observations, we include normal stress regularization following priors studies DeDontney et al. (2012); Xu et al. (2015b).

$\frac{d \tau}{d t}=-\frac{1}{t^{*}}\left(\tau-f \sigma_{N}\right)$
Table 1

Parameters description

\begin{tabular}{ll}
\hline Medium Material Properties & Value \\
\hline Shear Modulus $\mu$ & $32 \mathrm{GPa}$ \\
$S$ wave velocity $c_{s}$ & $3.464 \mathrm{~km} \cdot \mathrm{s}^{-1}$ \\
P wave velocity $c_{p}$ & $6.0 \mathrm{~km} \cdot \mathrm{s}^{-1}$ \\
Angle of Internal Friction $\psi$ & $30.96^{\circ}$ \\
Maximum Principle Stress direction $\theta_{p}$ & $19.33^{\circ}$ \\
\hline Fault constitutive Parameters & Value \\
\hline Static friction coefficient $\mu_{s}$ & 0.6 \\
Dynamic friction coefficient $\mu_{d}$ & 0.3 \\
Characteristic slip-weakening distance $d_{c}$ & $0.2 \mathrm{~m}$ \\
\hline Background Stress & Value \\
\hline Background Vertical Stress $\sigma_{y y}$ & $-50.0 \mathrm{MPa}$ \\
Background Horizontal Stress $\sigma_{x x}$ & $-100.0 \mathrm{MPa}$ \\
Background Shear Stress $\sigma_{x y}$ & $20.0 \mathrm{MPa}$ \\
\hline Domain Geometry & Value \\
\hline Reference length scale $L_{c}$ & $500 \mathrm{~m}$ \\
Length of the secondary faults $L_{f}$ & $1.0 L_{c}$ \\
Spacing between the secondary faults $L_{s}$ & $1.0 L_{c}$ \\
The off distance of the secondary fault from the main fault $L_{o}$ & $0.1 L_{c}$ \\
The angle between the secondary fault and the main fault $\theta_{f}$ & $30^{\circ}$ \\
Finite element cell size $h$ & $6.25 \mathrm{~m}$ \\
\hline &
\end{tabular}

where the shear strength $\tau$ evolves over a finite time scale $\left(t^{*}\right)$. $t^{*}$ was taken to be $2 \Delta x / c_{s}$, which is several times larger than the stable time step. Here $\Delta x$ is the mesh size.

\subsubsection{Geometry}

We consider our fault system to exist in an infinite medium. A planar horizontal main fault is placed in the middle of the domain with secondary fault branches explicitly modeled as shown in Fig. 1(a). The main fault is right lateral and the secondary faults are placed on one side of the fault (on the tension side) starting at a distance $L_{a}$ from the nucleation zone. This minimizes the effect of these secondary branches on the rupture nucleation. The secondary faults are distributed over a finite length along the main fault and not throughout the whole fault length so that we could explore features of the main fault rupture after it exits the region with the branches. The angle between the secondary faults and main fault is assumed to be $\theta_{f}$. The secondary faults have constant spacing $L_{S}$ along the fault strike. The length of each secondary fault is $L_{f}$. Vertically, the secondary fault branches are placed a small distance $L_{o}$ away from the main fault. We note that other approaches may be used to handle the triple junction between the branch fault and the main fault without having to enforce this shift. This may be accomplished by manipulating the kinematics of the split nodes at the junction through either retaining the continuity of the main fault only, or the continuity of the branch fault only, or by assuming that neither fault is continuous and having only one node at the triple junction point as described in DeDontney et al. (2012). The effect of the various modeling assumptions will be examined in future work. We limit the FEM discretization to a domain of length $L$ and width $W_{H}$. The length $L$ is taken to be $100 L_{c}$. The width $W_{H}$ is much smaller than the length $L$. The domain width $W_{H}$ is determined by the length of secondary branches and is taken to be $4 L_{c}$ to ensure that the FEM domain contains the complex fault geometry. All parameters are listed in Table 1.

\subsubsection{Initial and boundary condition}

We assume the domain is in static equilibrium at time $t=0$. We consistently resolve the normal stress $\sigma_{N}$ and tangential stress $\tau$ on all the faults from the background stress $\sigma_{x x}, \sigma_{y y}$ and $\sigma_{x y}$ 


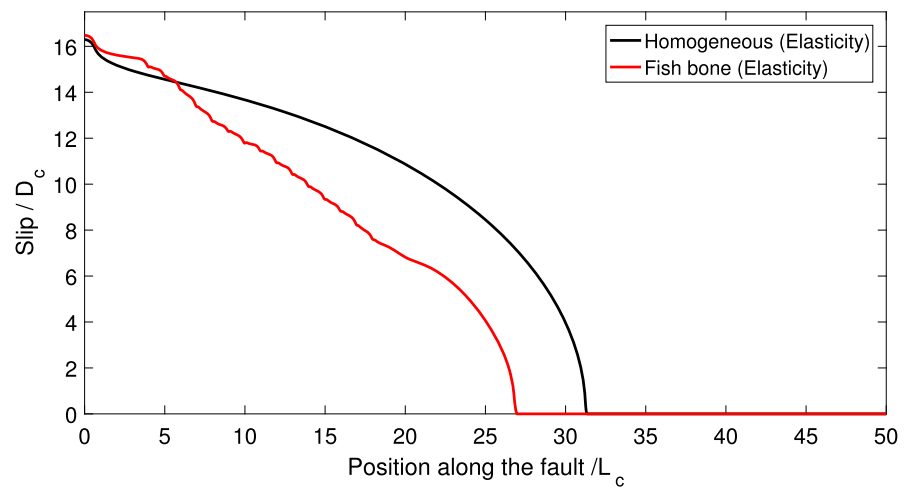

(a)

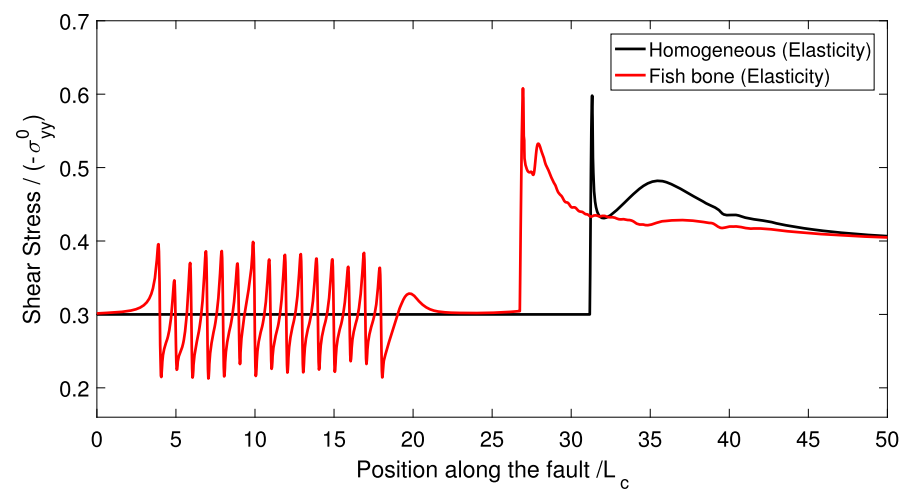

(c)

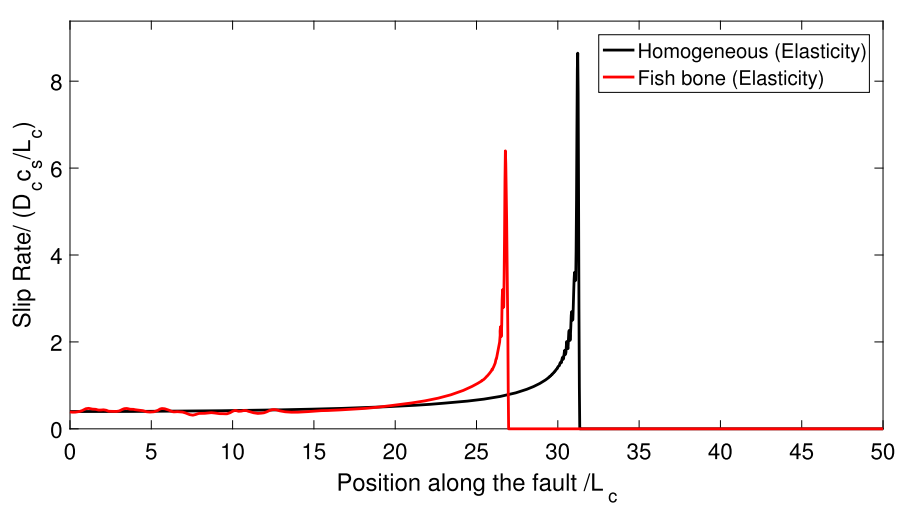

(b)

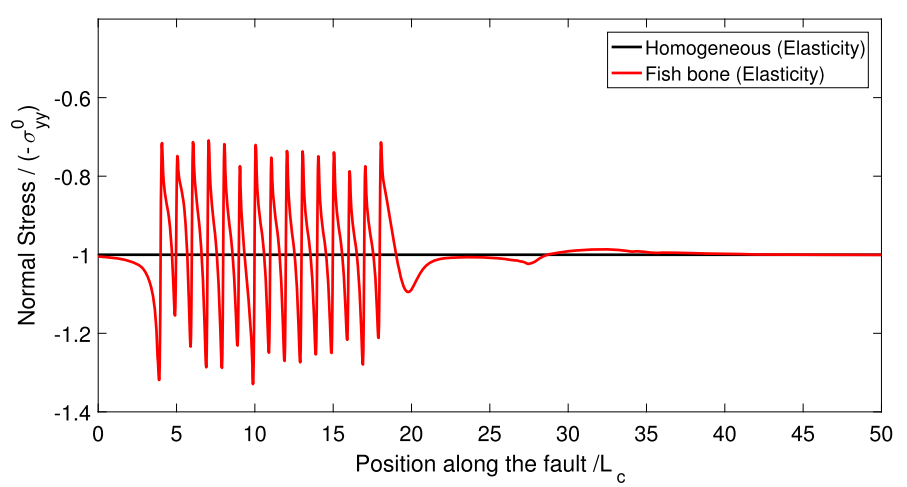

(d)

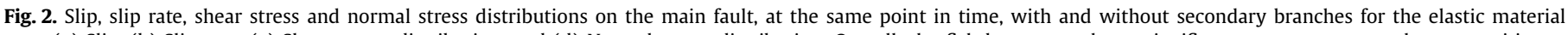

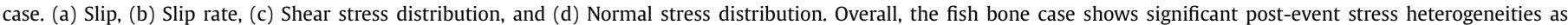

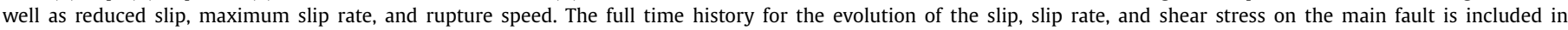
Supplementary Material 1.

using Eq. (12).

$$
\begin{aligned}
\sigma_{N} & =\sigma_{x x} \sin ^{2} \theta+\sigma_{y y} \cos ^{2} \theta-2 \tau_{x y} \sin \theta \cos \theta \\
\tau & =\sigma_{x x} \sin \theta \cos \theta-\sigma_{y y} \sin \theta \cos \theta+\tau_{x y}\left(\cos ^{2} \theta-\sin ^{2} \theta\right)
\end{aligned}
$$

where $\theta$ is the angle between secondary faults and the horizontal direction. We nucleate the rupture by overstressing the fault beyond the static friction strength over a localized region in its center with a width $L_{c}$. For the medium with elasto-plastic material, we apply a smooth nucleation approach. We use a union of hyperbolic tangent function to smoothly approximate an overstressing region width of $L_{c}$ to avoid stress concentration from the edges of the nucleation zone. The overstressing region stress level starts at 90 percent of the fault strength and gradually increases over a period of time to reach the fault strength stress level. Other nucleation approaches could also be used such as using consistent initial slip and slip rate profile extracted from quasidynamic simulations for the nucleation process on a planar fault Liu and Lapusta (2008).

From Andrews (1976); Das and Aki (1977), the relative strength parameter is defined as $S=\left(\tau_{s}-\tau\right) /\left(\tau-\tau_{d}\right)$, which quantifies the closeness of initial stress to failure relative to the stress drop. For this study, we are considering background stress conditions which correspond to strength parameter $S=2$ on the main fault. Thus, the ambient stress conditions favor sub-Rayleigh rupture propagation on the main fault.

\section{Results}

To normalize our results, we adopt the following dimensionless quantities for length, time, slip, slip rate, and stress:
- Length, $x^{*}=x / L_{c}$.

- Time, $t^{*}=t c_{s} / L_{c}$.

- Slip, $D^{*}=D / D_{c}$.

- Slip rate, $V^{*}=V L_{c} /\left(D_{c} c_{s}\right)$.

- Stress, $\sigma_{i j}^{*}=\sigma_{i j} /\left(-\sigma_{y y}^{0}\right)$.

\subsection{Elastic domain}

Fig. 2 compares several rupture metrics on the main fault plane with and without the short branches. The short branches lead to a reduction in the peak slip rate as well as the accumulated slip on the main fault plane. This may be explained by the fact that when the short branches are activated, the frictional slip on these secondary features contributes to the total energy dissipation leading to reduced slip and slip rate. The increased energy dissipation in the presence of the secondary branches also slows the rupture on the main fault and decreases the rupture propagation speed at least within the fish bone region. However, there is a slight increase in the slip near the center of the main fault (around $x^{*}=0$ ) for the case with the short branches. The initiation and arrest of ruptures on the secondary branches lead to the generation of seismic signals that are reflected back on the main fault leading to ripples in the slip rate profile that propagate backward (See Video 1 from Supplementary Material) and accumulate more slip away from the rupture tip that would not have been generated in the homogeneous medium case. The reduction in slip rate and rupture speed due to increased energy dissipation has also been previously observed in models with off-fault energy dissipation using plasticity Templeton and Rice (2008) or continuum 


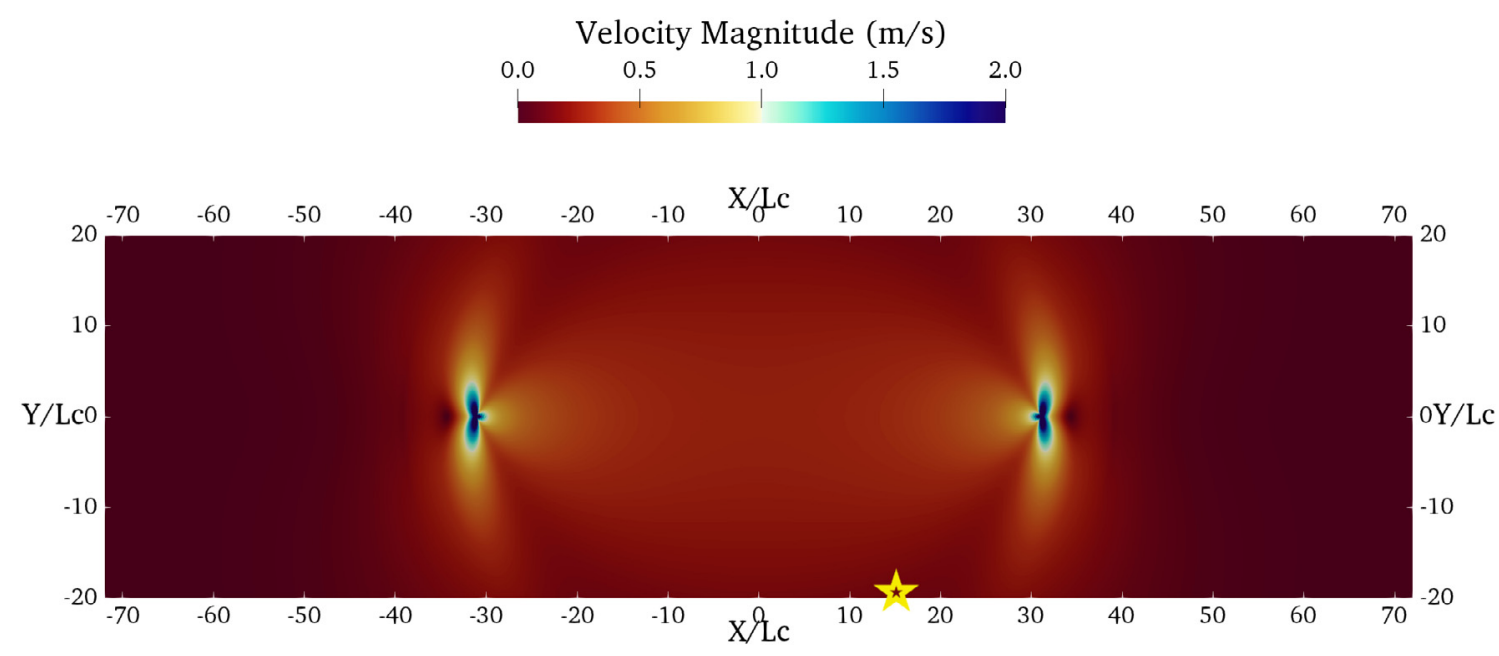

(a)

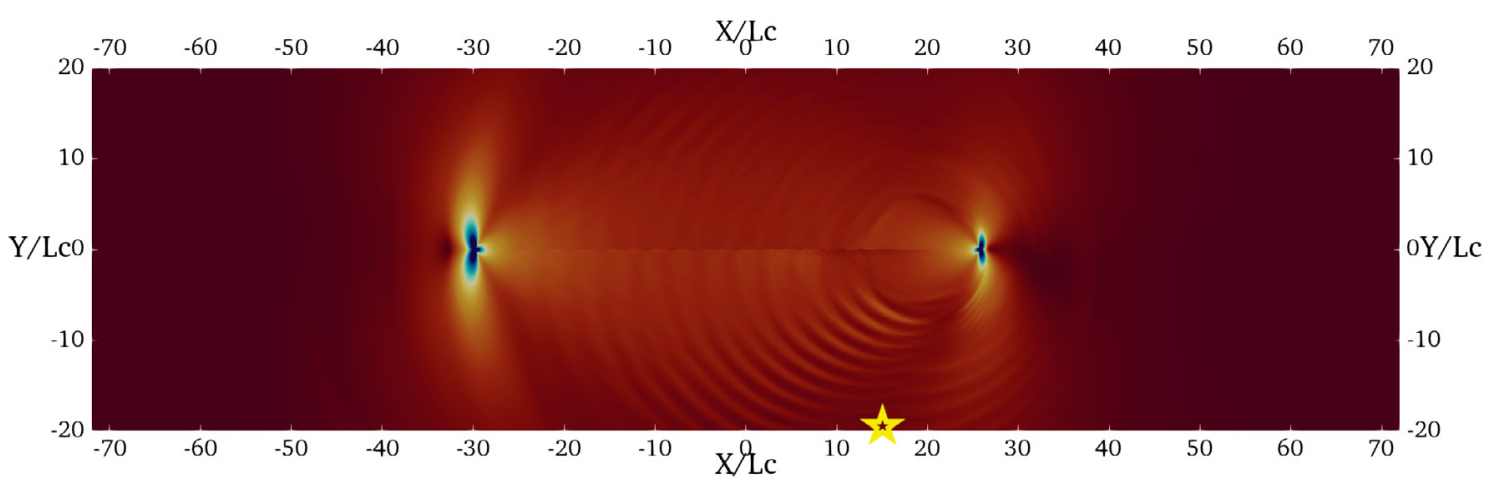

(b)

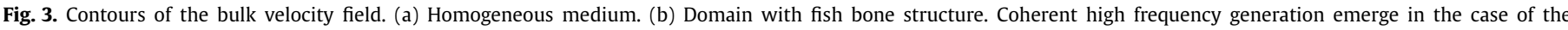

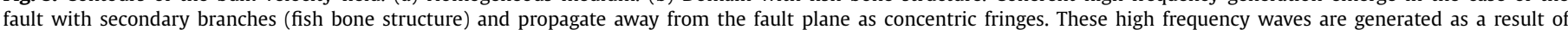

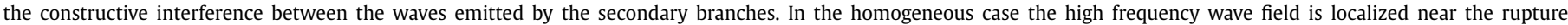

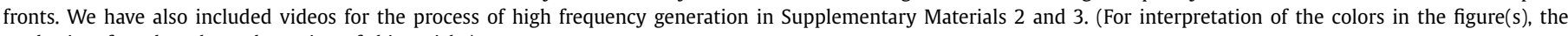
reader is referred to the web version of this article.)

damage theories Bhat et al. (2012). The backward propagating ripples, however, is a consequence of the geometric complexity of the model.

The secondary faults have a significant effect on the postrupture stress distribution. Fig. 2(c) and 2(d) show that both the shear and normal stress exhibit strong spatial heterogeneities within the fish bone region after the passage of the rupture front. These strong heterogeneities are absent in the homogeneous medium case. The activation and arrest of slip on the secondary branches lead to the development of normal and shear stress concentrations at their ends which load the main fault nonuniformly. These stress fluctuations lead to both stress increase as well as reduction in both of the normal and shear stress components. In particular, the normal stress is reduced to $70 \%$ of its original value at some locations. This may suggest that some configurations of the secondary branches may even lead to fault opening, although we have not observed this yet in the cases we investigated. Furthermore, the shear stress drops to $50 \%$ of its corresponding value in the homogeneous case at several points. This is also indicative that geometric complexities may potentially lead to the reversal of the shear stress sense if they cause large enough shear stress fluctuations. This pattern of stress fluctuations on the main fault may be predicted qualitatively using Linear Elastic Fracture Mechanics (LEFM) as has been done in a previous study of dynamic rupture with a single backthrust branch fault Xu et al. (2015b). We present an example of such calculations in Appendix A

Another major result in this paper is the influence of secondary branches on the high-frequency generation in the bulk. Fig. 3 shows the near-field particle velocity for both cases with and without the secondary branches. For the homogeneous medium, the wave field is smooth almost everywhere with concentration of high frequencies neat the rupture tips. On the other hand, for the medium with branches, we observe coherent wave fronts that are propagating away from the tips and spaced apart periodically, consistent with the periodic distribution of the secondary branches. These coherent fronts are generated due to the constructive interference of seismic radiation from the secondary faults. We have also included videos for the process of high frequency generation in Supplementary Materials 2 and 3.

To demonstrate the enhanced generation of high frequencies for the case with the fish bone structure, we plot in Fig. 4 the faultparallel and fault-normal components of the velocity at a station located $20 L_{c}$ from the main fault and represented by the star in Fig. 3. Both components of the velocity show high frequency fluctuations in the case of the fault with branches compared to the homogeneous case. The acceleration spectra plotted in Fig. 4(c) further prove this point. The fault with small branches has a spectrum that is richer in high-frequency content and furthermore shows an 


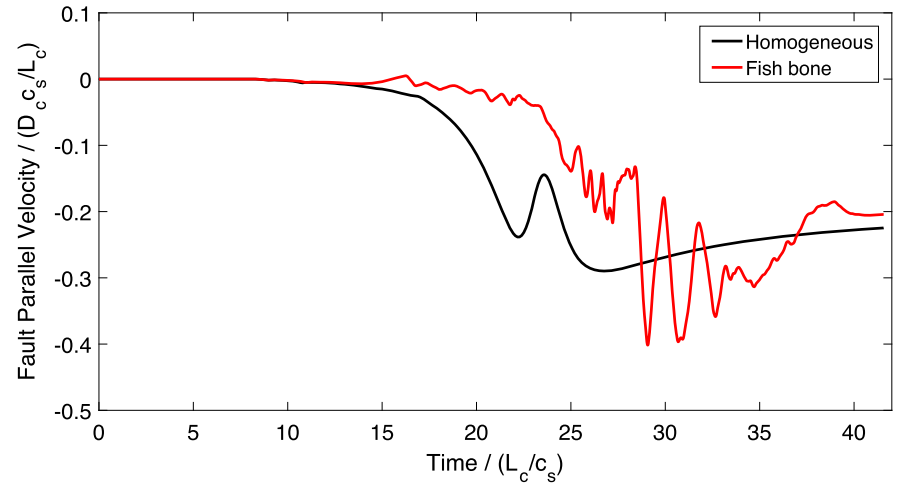

(a)

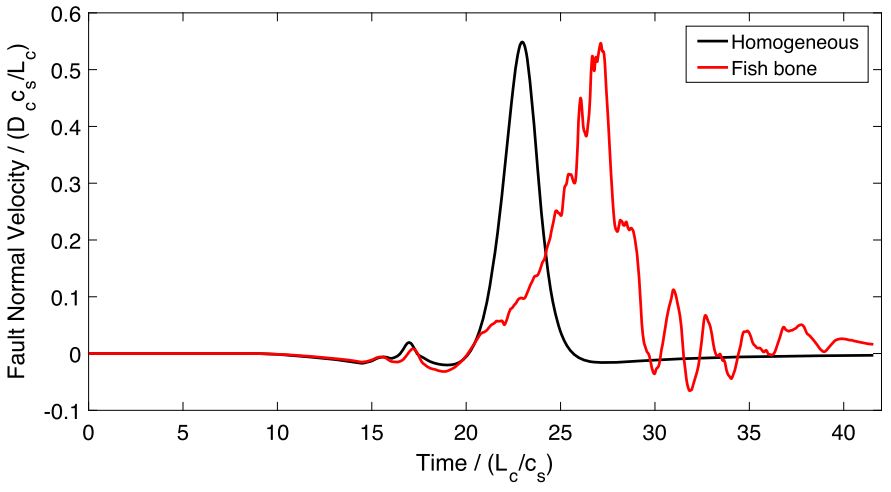

(b)

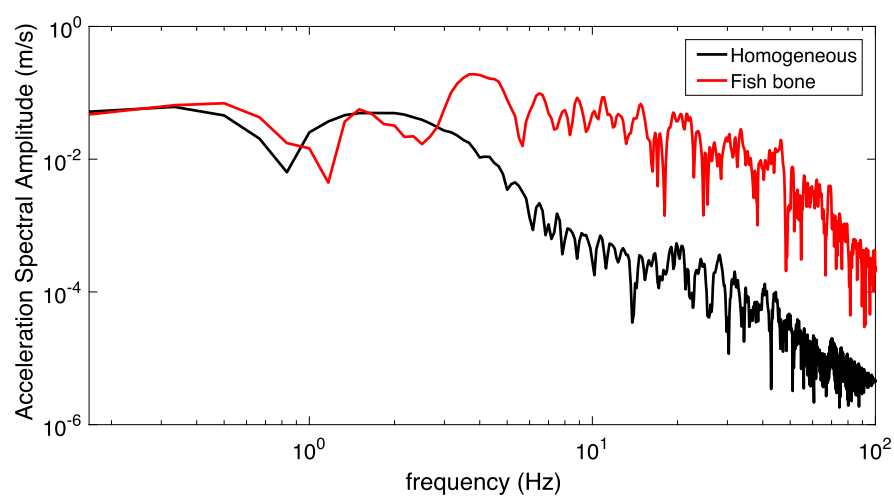

(c)

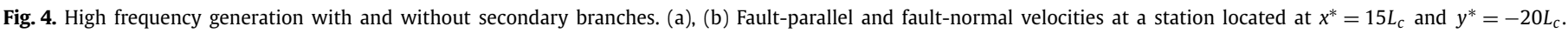
(c) Fault-normal acceleration spectral amplitude at station $x^{*}=15 L_{c}$ and $y^{*}=-20 L_{c}$.

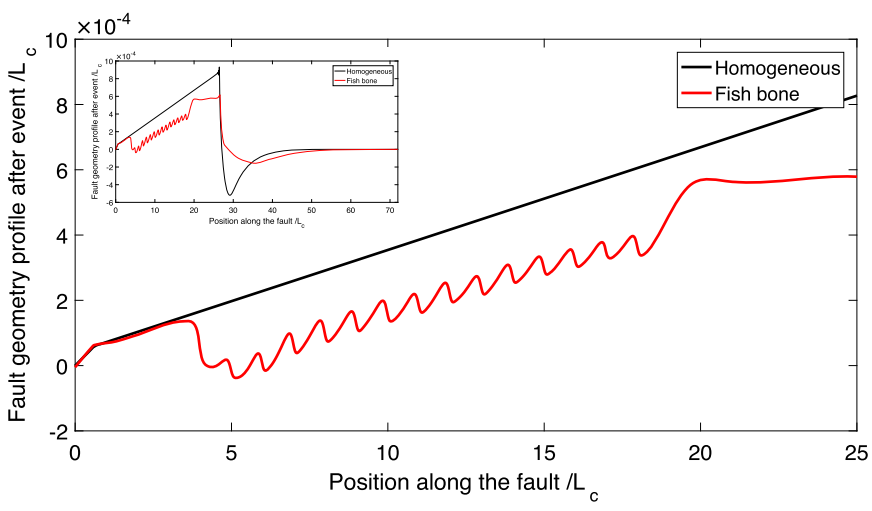

Fig. 5. Normal displacement distribution with and without secondary branches. The insert figure shows the whole distribution along the full half length of the fault. The secondary faults cause periodic undulations in the main fault profile.

almost flat spectrum in the frequency range $2-20 \mathrm{~Hz}$. This is consistent with observations in Chen (1995); Wald and Heaton (1994) and similar to the results from dynamic rupture simulation on rough faults Dunham et al. (2011b). This suggests that small scale fault branches may be a candidate for explaining near field radiation characteristics of active faults.

Another effect of the secondary faults is shown in Fig. 5 which illustrates the distribution of the normal displacement of the main fault plane. For the homogeneous medium, the fault plane simply rotates. The existence of the secondary branches, however, leads to the development of undulations in the fault plane profile as shown in Fig. 5. The stress concentrations corresponding to the secondary faults, load the fault in the normal direction and promote repeated peaks in its vertical profile near the locations where the secondary branches are positioned. While the magnitude of these undulations is small, they may contribute, over several cycles, to the evolution of the main fault roughness.

To gain further insight into the dynamics of the branch faults, we show in Fig. 6 the time evolution of the slip, slip rate, and the rupture speed on one of the secondary faults (the first branch). The secondary fault is triggered dynamically by the main fault rupture as it approaches the branch tip leading to a rapid increase in slip rate and slip over a segment of the branch that is closest to the main fault. As stated in Section 2, the background stress favors a sub-Rayleigh rupture propagation on the main fault $(S=2)$. However, this is not the case for the secondary faults which are loaded dynamically from the propagating rupture on the main fault in addition to the loading from the background stress field. The insert in Fig. 6(a) shows the rupture tip position along the secondary fault versus time, and it suggests that the secondary fault fails in a supershear mode. Interestingly, the background strength parameter for the secondary faults in this case is $S=-8.12$ (See Table B.1 in Appendix B) corresponding to an initially resolved shear stress that is lower than the dynamic frictional stress. Thus, this result suggests that even though the far field background stress favors a sub-Rayleigh rupture propagation on the fault system, the small scale branching faults may fail differently when excited dynamically. This may potentially have important implications for seismic hazard from complex fault zones.

We have also carried out a set of simulations to explore the effect of various geometric features of the fish bone structure, including secondary faults length, orientation, and spacing, on the dynamics of the main fault rupture. The results are summarized in Appendix B indicating that many of the features reported for the default case still hold and suggesting that the interplay between 


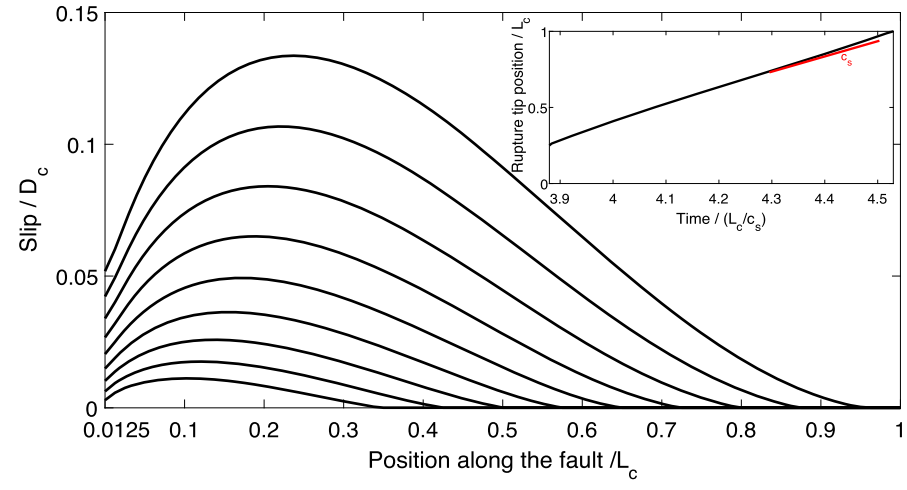

(a)

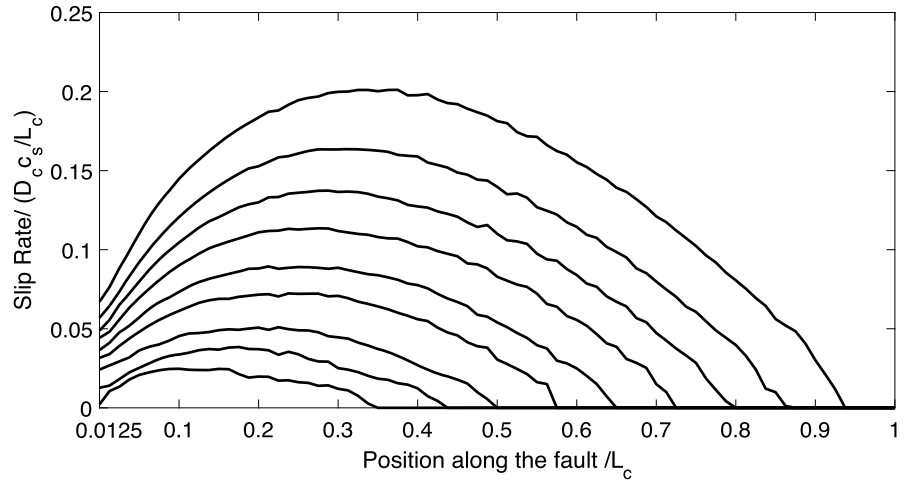

(b)

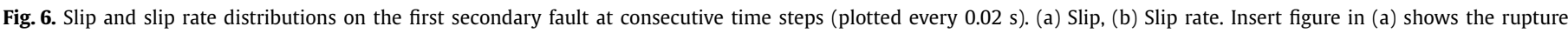

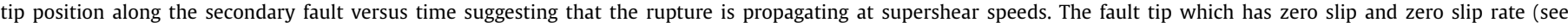
Fig. 1(c)) is excluded from the plot for clarity.

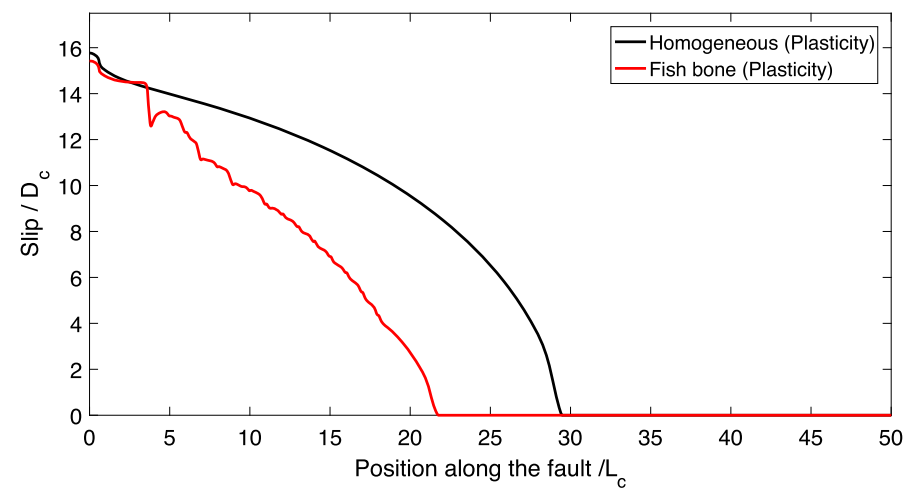

(a)

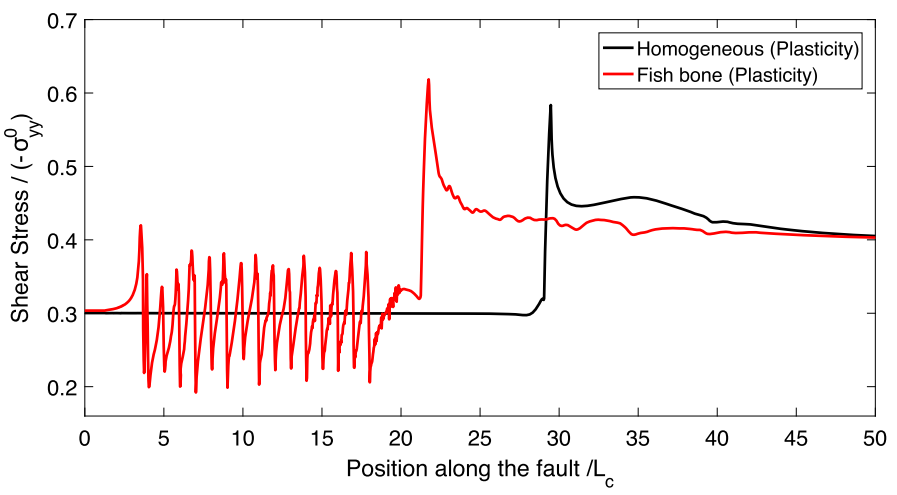

(c)

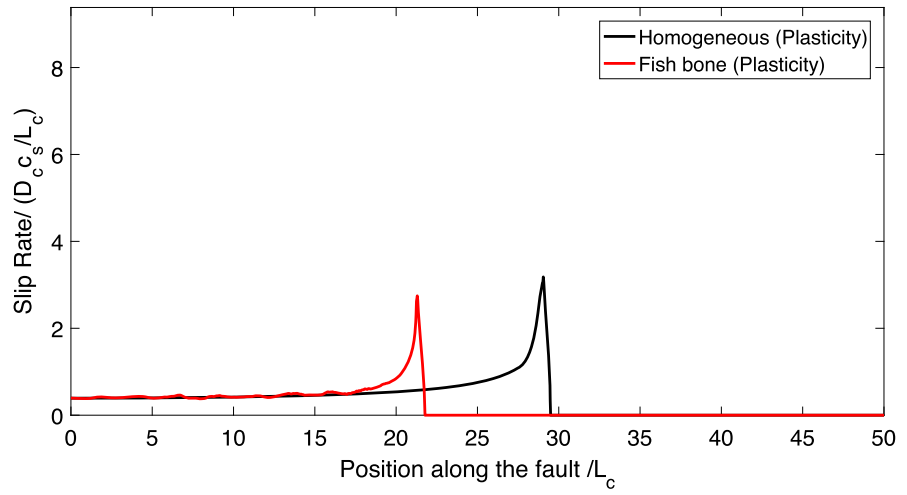

(b)

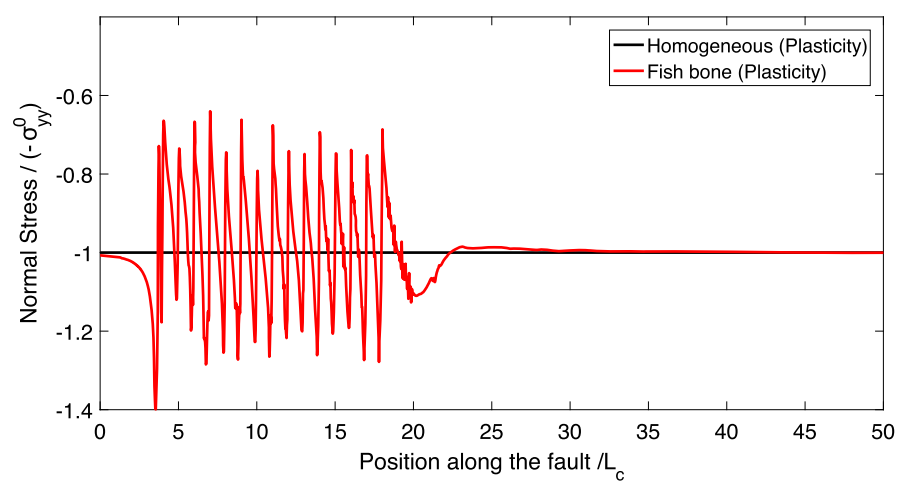

(d)

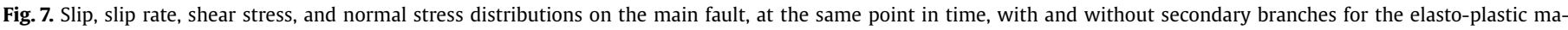

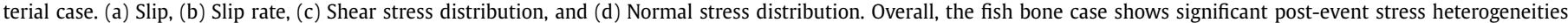
as well as reduced slip, maximum slip rate, and rupture speed. The values of slip and maximum slip rate in the elasto-plastic case are lower than the elastic case.

dynamics and geometry enriches the rupture complexity. This will be further investigated in a future study.

\subsection{Elasto-plastic domain}

To account for additional energy dissipation mechanisms at a scale smaller than the scale of the secondary branches that we haven't explicitly modeled, we consider the possibility of inelastic strain generation using an elasto-plastic material model. Since we have only considered one level of the secondary branches, the plasticity model may be used as a proxy for small scale damage that is randomly distributed and arising from microcracks or dislocation movement at nano or micro scale. Drucker-Prager plasticity is used as described in Section 2.

Fig. 7 compares several rupture metrics on the main fault plane with and without the short branches but in the presence of offfault plasticity. In this case, the rupture may generate off-fault plastic strain if the Drucker-Prager yield criterion is met. Consistent with the elastic case, the short branches also lead to a reduction in the peak slip rate as well as the accumulated slip on the main fault plane. The frictional slip on the secondary branches contributes to the total energy dissipation leading to reduced slip, slip 


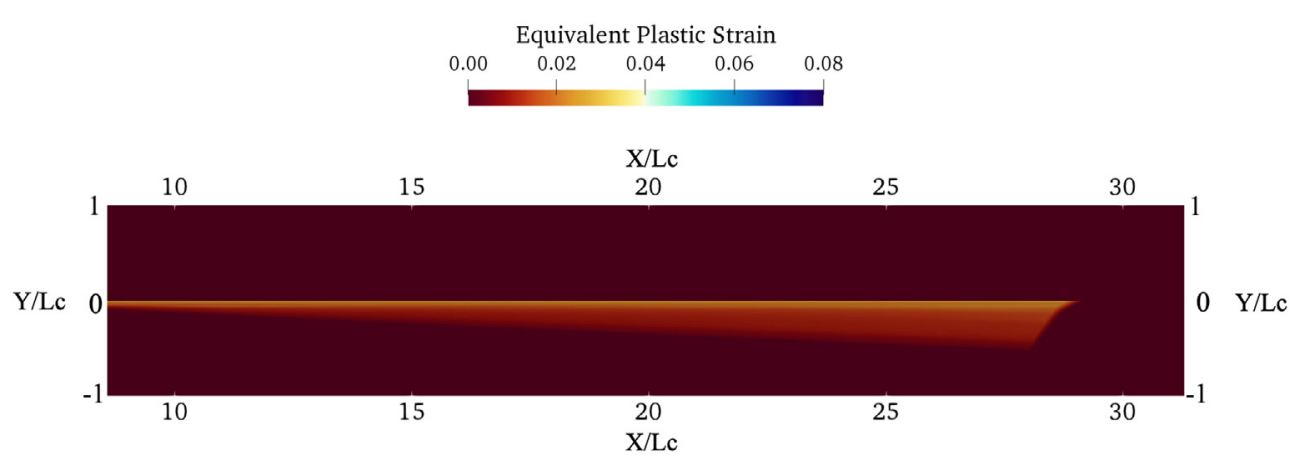

(a)

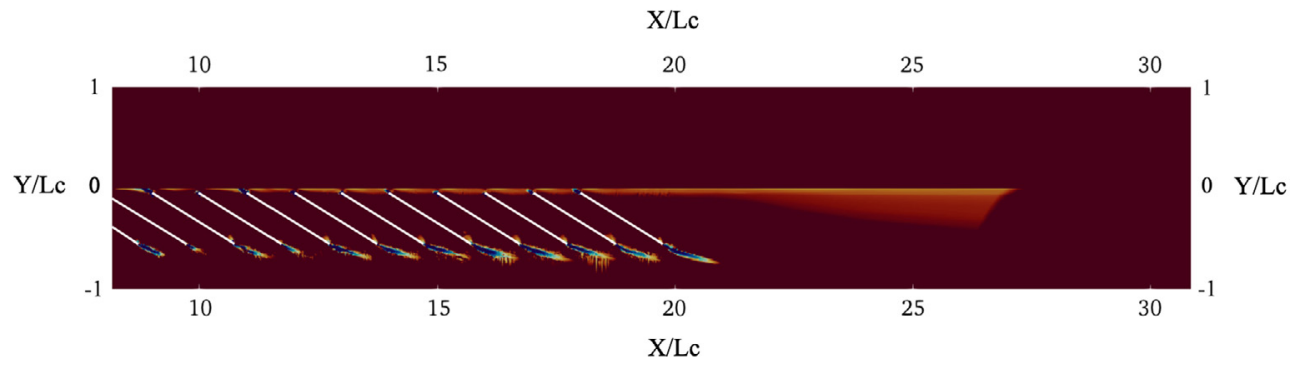

(b)

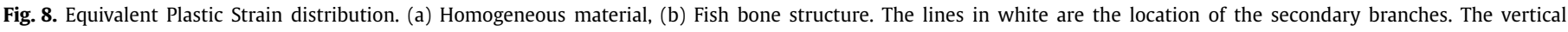
dimension is exaggerated for visualization purpose.

rate, and rupture propagation speed. However, unlike in the elastic case, there is no slight increase in the slip near the center of the main fault (around $x^{*}=0$ ) for the case with the short branches. Plasticity, which acts as an additional energy sink on its own, has suppressed the backward propagating ripples and greatly reduced their effect. Overall, the slip, the slip rate, and the rupture speed are all lower in this case compared to the case of rupture propagation in an elastic medium.

The effect of the secondary faults on the post-rupture stress distribution persists even with plasticity. Fig. 7(c) and 7(d) show that both the shear and normal stress exhibit strong spatial heterogeneities within the fish bone region after the passage of the rupture front. These strong heterogeneities are absent in the homogeneous medium case with off-fault plasticity. The activation and arrest of slip on the secondary branches leads to the development of normal and shear stress concentrations at their ends which load the main fault nonuniformly. These stress fluctuations lead to both stress increase as well as reduction in both of the normal and shear stress components and the amplitude of the fluctuations are very similar to those generated in the elastic case indicating that they are unaffected by plasticity.

The secondary branches, as pre-existing damage features, have strong influence on the off-fault plastic strain distribution as shown in Fig. 8. While in the homogeneous case, the plastic strain distribution has the characteristic fan-like shape consistent with previous studies Dunham et al. (2011a,b); Templeton and Rice (2008), the plastic strain distribution is increasingly non-uniform due to the presence of the short branches. In particular, the spatial extent of the off-fault plasticity in the vicinity of the main fault is greatly reduced within the region that hosts the short branches. Furthermore, the short branches seem to have little or no plastic strain accumulation, suggesting that what should have been bulk plastic strain has collapsed in the form of localized slip along the short secondary fault. However, there is a large increase in the plastic strain accumulation at the ends of the short branches due to the abrupt arrest of the slip and the associated stress concentra- tion. Namely, there is a concentration in plastic strain in the region between the secondary branch tip and the main fault suggesting that even if the branch is not directly connected to the main fault, this region will be severely damaged. Furthermore, there is another region of plastic strain concentration at the far end of the secondary fault. This region also does not extend along the strike of the secondary branches but is slightly bent in another direction suggesting a possible growth plane for the secondary faults if they are allowed to extend.

\subsection{Rupture characteristics with and without plasticity}

Fig. 9(a) shows the rupture tip position versus time for four cases: the homogeneous medium with and without plasticity, and the fish bone structure with and without plasticity. The slope of these curves gives the rupture propagation speed for each case. The existence of the secondary branches significantly reduces the rupture speed compared to the homogeneous case. The rupture propagation speed generally decreases with off-fault plastic dissipation. The rupture propagates the slowest on the main fault for the case with fish bone structure in elasto-plastic medium. An unexpected observation is that with the existence of the secondary branches, the rupture may temporarily travel faster than the homogeneous case at first and then decelerate (See insert of Fig. 9(a)). This may be explained by the fact that initially the rupture speed on the main fault is small, and that when these secondary branches are activated, they generate waves that may constructively interfere with the main rupture tip, channel energy to this tip, and promote its transient acceleration. As the main rupture accelerates further, this effect is diminished and the secondary faults act primarily as energy sinks, increasing the overall energy dissipation and decelerating the main fault rupture propagation. Once the rupture tip on the main fault moves beyond the fish bone region, it accelerates further approaching the propagation speed of the rupture in the homogeneous case with and without plasticity respectively. 


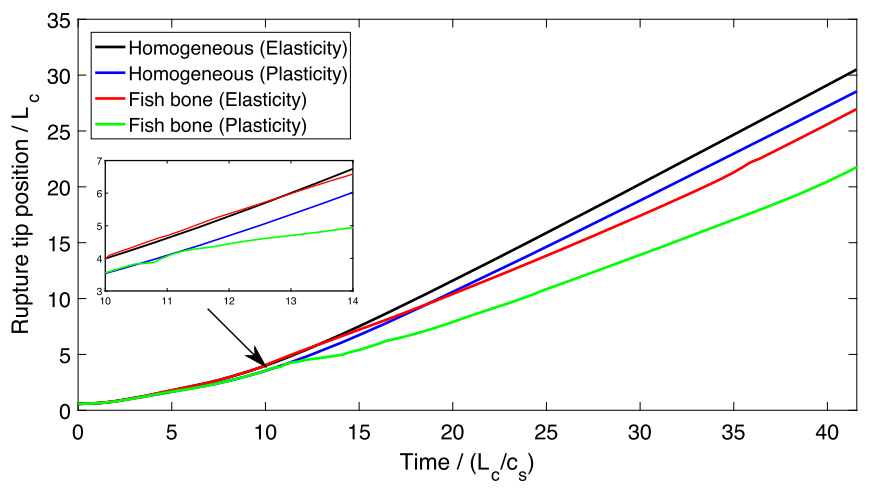

(a)

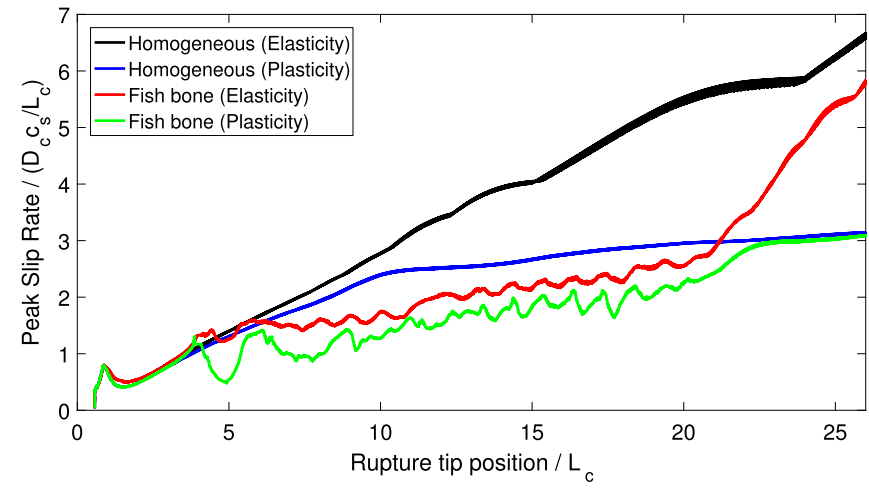

(b)

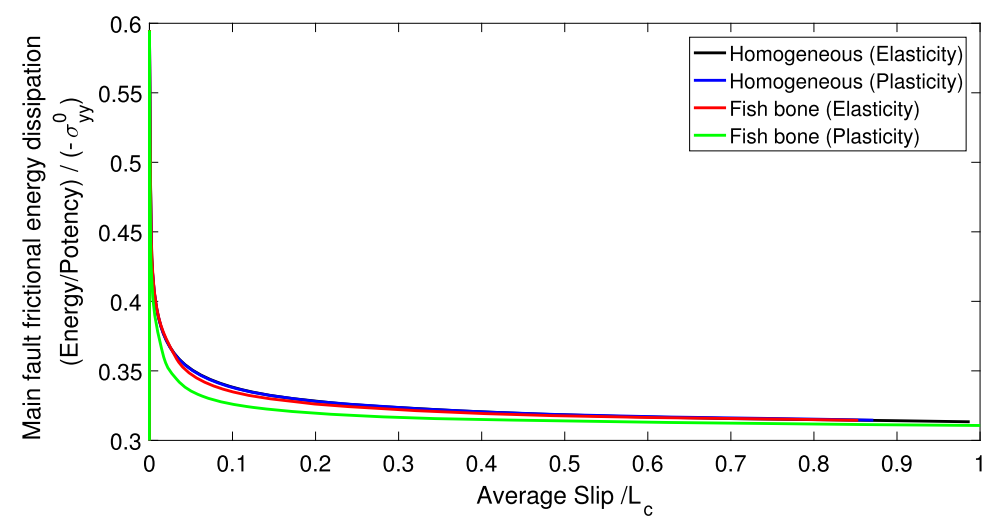

(c)

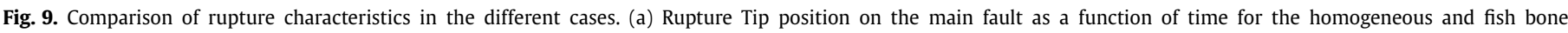

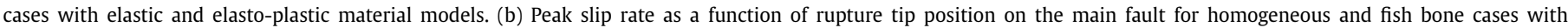

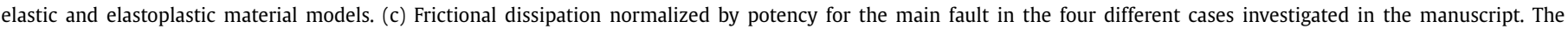

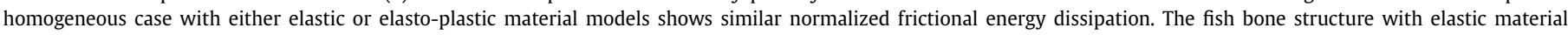

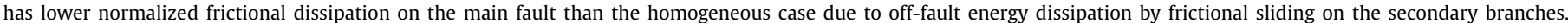

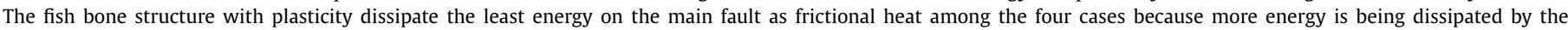
localized plastic deformation at the tips of the secondary faults.

Fig. 9(b) shows the maximum slip rate versus rupture tip position for the different cases. The secondary branches lead to a significant reduction in the peak slip rate on the main fault. Cases with off-fault plasticity also show a reduction in the peak slip rate compared to the elastic case. The existence of secondary branches also leads to high-frequency oscillations in the peak slip rate as the rupture propagates, indicative of enhanced radiation efficiency and high-frequency generation. After the rupture on the main fault has propagated beyond the region with the fish bone architecture, the peak slip rate increases and approaches the peak slip rate values for rupture propagation in the homogeneous medium.

Fig. 9(c) shows the main fault frictional energy dissipation normalized by the potency at each time step versus the average slip for the fish bone case and the homogeneous case with and without plasticity. The frictional dissipation is calculated by integrating the product of the frictional stress and the slip rate over the fault length and over time $E_{f}=\int_{0}^{t}\left(\int \tau_{f} \dot{D} d a\right) d t^{\prime}$. The potency is defined as the integral of the slip over the fault domain $P=\int D d a$. The frictional dissipation normalized by the potency gives a stress-like quantity which may be taken indicative of an average frictional strength on the fault. Thus, the plots shown in Fig. 9(c) may be considered as modified effective slip-weakening laws for the fault as a whole. The homogeneous cases with and without plasticity have relatively similar effective stress-slip response. This is because the energy dissipated by off-fault plasticity is smaller than 0.1 percent of the frictional dissipation. Interestingly, the fish bone structure case with plasticity shows the least amount of frictional energy dissipation on the main fault of the four cases. This may be attributed to the other energy dissipation avenues that exist due to the combination of off-fault plasticity and frictional slip on the additional surfaces of the secondary faults. In particular, in the complex fish bone structure, the stress tends to be concentrated at the ends of the secondary faults leading to higher concentration of the plastic strain in this region. This increases the contribution to off-fault energy dissipation on the expense of the energy dissipation by frictional sliding on the main fault.

\section{Discussion}

Earthquake ruptures are nonlinear multiscale phenomena. A fundamental challenge in earthquake source physics is to resolve this vast range of scales. In this paper we have focused on resolving the influence of one of the intermediate spatial scales, namely small scale fault branches, on the rupture dynamics of a single event. These branches are characterized as being small scale since their length is of the order of the reference length scale for nucleation in mature faults.

Our investigation of the effect of explicitly represented small scale branches on rupture dynamics reveals several results that are consistent with the more conventional method of modeling small scale damage as an effective elasto-plastic or continuum damage constitutive relation. For example, slip on these secondary faults increases the overall energy dissipation leading to a reduction in the accumulated slip, maximum slip rate, and rupture propagation 
speed on the main fault. However, explicit representation of these anisotropic pre-existing slip planes also lead to some novel insights that may not be captured by continuum plasticity models.

For example, the interaction of the main rupture with the short branches leads to strong heterogeneities in the final normal and shear stress distributions. These stress fluctuations may potentially lead to fault opening or reversal in the sign of the shear stress on the main fault, although this has not been observed within the parameter range explored in this paper. Interestingly, these stress heterogeneities due to the existence of the secondary branches persist even in the presence of elasto-plastic material response. They do not get smeared or homogenized. The nonuniform stress distribution left over after the seismic event may influence the nucleation, propagation, and arrest of future seismic events. Furthermore, the secondary branches may also act as potential nucleation sites for future ruptures, that do not lie directly on the main fault, but may potentially jump over to its plane. Thus, there is significant potential that this model may form a basis for earthquake complexity.

Moreover, explicit representation of the secondary branches suggest that these features may contribute significantly to the near field high frequency generation. The constructive interference between the seismic radiation from the secondary faults lead to coherent high-frequency generation in the bulk that is strongly correlated to the geometric distribution of the secondary branches. Furthermore, we demonstrated that the near-field acceleration spectrum in the presence of secondary faults is almost flat in the range of 2-20 Hz. This feature has been widely documented in observations Chen (1995); Wald and Heaton (1994). It is also similar to what Dunham et al. (2011b) have observed in dynamic rupture simulations on rough faults. This suggests that complex geometric features, other than fault roughness, such as secondary short branches, may lead to similar coherent high frequency generation patterns.

The secondary faults may act as both energy sinks and energy sources for the main fault. This apparently conflicting role may be explained as follows. As the main fault rupture propagates and the secondary faults are being activated, energy is being dissipated by slip on the secondary faults and therefore the branches act as energy sinks. In the meantime, as the secondary faults are activated there is dynamic stress drop on these faults. This generates seismic waves that may load the main fault favorably leading to a transient acceleration in the main fault rupture propagation speed or slip rate. Thus, in this limit, the secondary faults may act as energy source. However, this effect depends on the orientation of the secondary fault as well as the stage of the rupture on the main fault (e.g. its current rupture speed). For example, in Fig. 9(a) the transient increase in the rupture speed on the main fault happens in the early stages of the rupture where the speed is still lower than the limiting speed. Later in the rupture history, the effect of the secondary fault energy dissipation dominates over the transient dynamic stress transfer and the rupture speed on the main fault decreases.

Different mechanisms have been proposed for fault roughness evolution Ben-Zion and Sammis (2003); Brodsky et al. (2016). These mechanisms include fragmentation, wear, and healing. We have shown here that slip on secondary branches may lead to stress concentrations that load the main fault in a way that leads to undulations in the fault plane with a periodicity comparable to the spacing between the secondary branches. While the amplitude of these undulations is small, they may grow due to repeated ruptures, thus, providing an additional mechanism for fault plane roughness evolution on small scales.

In this paper, we have used linear slip-weakening as the fault constitutive model. The rate and state friction framework Dieterich (1979); Ruina (1983), however, has been successful in interpreting several lab and field observations. While the slip-weakening friction may not be a realistic representation of the fault physics, it is a useful mathematical model that may approximate rate and state friction response, without strong velocity weakening, with the appropriate choice of parameters. In future work, we plan to investigate our results in the framework of rate and state friction with dynamic weakening to explore the role of large dynamic stress drops as well as time dependent post-seismic deformation on the stress concentrations generated by the fish bone structure.

The recent models by Klinger et al. (2018) provide a pioneering step towards exploration of the influence of co-seismically evolving off-fault damage on rupture dynamics. The current paper complements these on-going efforts in the community and provides a step forward towards explicit inclusion of small scale physics in fault zone in the form of pre-existing anisotropic damage features. Continuum damage models and conventional plasticity algorithms are prone to numerical localization. In our case, we pre-define the secondary slip planes based on the background tectonic stress field. While this biases our choice for the fault plane orientations, our results are not mesh dependent. There is a need for development of computational algorithms that may nucleate and grow faults on the fly with minimum or no mesh dependency. Potential candidates include nonlocal damage and plasticity models Ma and Elbanna (2018); Preuss et al. (2019), extended finite element methods Liu and Borja (2009, 2013), and Discontinuous Galerkin scheme with adaptive mesh refinement Pelties et al. (2012, 2014).

In this paper, for modeling energy dissipation at scales smaller than the scale that is explicitly represented by the secondary branches, we adopted the rate-independent Drucker-Prager plasticity model. Without any regularization, the model is prone to artificial strain localization. While the stress concentration at the tips of the secondary branches is physical and necessitates a concentration in the plastic strain, a robust feature in our model that seems to persist at different resolutions, the orientation of the localization band shown in Fig. 8 around the tips of the fish bone structures may have a mesh-dependent ingredient. In the results presented here, the reported shear bands are several elements wide in some places but this does not entirely eliminate the mesh sensitivity. In future work, a rate-dependent plasticity model will be used such as rate-dependent Drucker-Prager plasticity model or rate sensitive Shear Transformation Zone theory Ma and Elbanna (2018) to avoid or limit the effects of any potential numerical artifacts.

In this paper we introduced an application of the recently developed hybrid method which attests to its potential for modeling dynamic rupture with high resolution fault zone physics. While explicit representation of short branches is a start, other candidate applications are also possible. For example, we may use the hybrid method to model strain localization and shear band evolution within the gouge region Ma and Elbanna (2018) while maintaining the influence of long range elastic stress transfer in the bulk. Another potential application is to model small-scale damage patterns, as has been done experimentally by Biegel et al. (2010) to study the transient and steady-state effect of damage patterns on the rupture dynamic. These problems are too challenging for the traditional domain-based numerical schemes but the efficient domain truncation using the hybrid scheme may make them more doable.

Future extensions of this work may include expanding the parametric study initiated here to include nonuniform spacing, orientation, and length of the secondary faults. The ultimate goal would be to use the hybrid scheme to model earthquake cycles in complex fault zone structures bridging both seismic and aseismic episodes and enabling the interplay between dynamics, stress evolution, and geometry to understand the underpinnings of earthquake complexity. 


\section{Conclusion}

In this paper, we apply our recently developed hybrid numerical scheme to investigate the influence of explicitly represented small scale branches on rupture dynamics. The main conclusions may be summarized as follows:

- The secondary faults increase the overall energy dissipation leading to a reduction in the slip, peak slip rate, and rupture propagation on the main fault.

- The activation of the secondary faults may lead to backward propagating ripples in the slip rate that increases slip far from the rupture tip.

- Rupture activation, propagation, and arrest on the secondary branches lead to a strongly heterogeneous normal and shear stress field on the main fault.

- The interaction of the seismic waves generated by the secondary branches promotes high-frequency generation and generate high-frequency fluctuations on the computed seismograms.

- The secondary branches lead to the evolution of normal undulations in the main fault strike.

\section{Acknowledgement}

The authors are grateful for comments from the reviewers which helped improve the manuscript. The authors acknowledge support from the Center for Geologic Storage of $\mathrm{CO} 2$, an Energy Fronter Research Center funded by the U.S. Department of Energy (DOE), Office of Science, Basic Energy Sciences (BES), under Award \#DE-SC0C12504, for development of the computational method. The authors also acknowledge support from the Southern California Earthquake Center through a collaborative agreement between NSF. Grant Number: EAR0529922 and USGS. Grant Number: 07HQAG0008 and the National Science Foundation CAREER award No. 1753249 for modeling complex fault zone structures. AE acknowledges additional support provided by the Department of Energy under Award Number DE-FE0031685 which facilitated conducting the parametric study.

\section{Appendix A. Linear elastic fracture mechanics analysis on the stress perturbation on the main fault}

Here we present an example calculation of using Linear Elastic Fracture Mechanics to predict the stress perturbation pattern on the main fault due to the presence of a secondary fault in its vicinity. We idealize the secondary fault as a Mode II finite length crack in an infinite domain. From Sun and Jin (2012), the stress distribution around the crack tip in this case is given by Eq. (A.1)

$$
\begin{aligned}
\sigma_{x x} & =-\frac{K_{I I}}{\sqrt{2 \pi r}} \sin \frac{\phi}{2}\left(2+\cos \frac{\phi}{2} \cos \frac{3 \phi}{2}\right) \\
\sigma_{y y} & =\frac{K_{I I}}{\sqrt{2 \pi r}} \sin \frac{\phi}{2} \cos \frac{\phi}{2} \cos \frac{3 \phi}{2} \\
\sigma_{x y} & =\frac{K_{I I}}{\sqrt{2 \pi r}} \cos \frac{\phi}{2}\left(1-\sin \frac{\phi}{2} \sin \frac{3 \phi}{2}\right)
\end{aligned}
$$

where $K_{I I}$ is the stress intensity factor for Mode II fracture. $\phi$ and $r$ are the angle and radius in the polar coordinate system. The geometry model is defined as shown in Fig. A.1.

From Eq. (A.1), we compute the stress tensor $\sigma$ from which we may compute the normal traction component $\sigma_{n}$ and the tangential component $\tau$ on the main fault as given by Eq. (A.2):

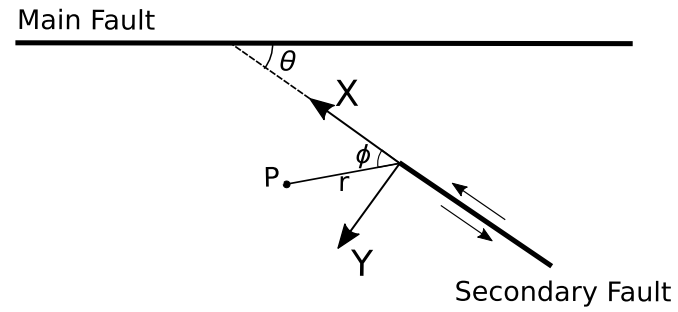

Fig. A.1. Model geometry setup for Linear Elastic Fracture Mechanics analysis. The origin of the coordinate system is set at the near end of the secondary fault. The polar coordinates are defined with radius $r$ and angle $\phi$. The angle between the main fault and the secondary fault is $\theta$. The shear on the secondary fault is left lateral.

$$
\begin{aligned}
\mathbf{T} & =\boldsymbol{\sigma} \mathbf{n} \\
\sigma_{N} & =\mathbf{T} \cdot \mathbf{n} \\
\tau & =\sqrt{|\mathbf{T}|^{2}-\sigma_{N}}
\end{aligned}
$$

Where $\mathbf{n}$ is the vector normal to plane of the main fault. The results are plotted in Fig. A.2, and give a pattern for the stress perturbation expected from a branch that qualitatively agrees with the numerical results shown in the paper.

\section{Appendix B. Parametric study for the elastic case}

In order to explore the effect of the secondary faults on the rupture characteristics of the main fault, we carried out a limited parametric study by varying some geometric properties of the secondary faults including length $L_{f}$, spacing $L_{s}$, and the angle with the main fault $\theta_{f}$.

\section{Effect of secondary fault length}

Fig. B.1 shows a snapshot of slip, slip rate, shear stress, and normal stress distribution on the main fault at a given instant of time. We examine three cases of secondary fault length $L_{f}=L_{c}$; $4 L_{c} ; 6 L_{c}$, while keeping all the other parameters the same as in the default case. With increased length of the secondary faults, the rupture speed on the main fault decreases as well as the maximum slip rate as shown in Fig. B.1(b). However, the oscillations in the slip rate, shown in the insert in Fig. B.1(b), increase with increasing the secondary faults length. Furthermore, Fig. B.1(c) and Fig. B.1(d) show that longer secondary faults promote a more complex pattern in the shear and normal stress perturbations. In particular, not all stress peaks or troughs have the same amplitude. This is because with longer secondary faults, slip is not necessarily accumulated through the whole length of each fault suggesting that some secondary faults may accumulate less slip or their rupture may stop before reaching the far end of the secondary fault. Fig. B.2 shows the distribution of maximum slip on the secondary faults for different secondary fault length. The results suggest that as the secondary fault length increases, a crack shielding effect emerge; the slip distribution along the secondary faults is non-uniform in the sense that as one secondary fault accumulates large slip, the following one or two accumulate smaller slip, but then comes another secondary fault with large slip, and the pattern continues. The non-uniformity in slip that increases as the secondary fault length increases, leads to non-monotonicity in the stress peaks on the main fault with some of the peaks smaller than others. This crack shielding-like phenomenon (sometimes also referred as stress shadowing phenomenon) has been observed both in the experimental work by Ngo et al. (2012) for tensile cracks as well as numerical simulation results using finite-discrete element method by Klinger et al. (2018) and other studies modeling spontaneous crack branching Ando and Yamashita (2007) and off-fault plasticity Templeton and Rice (2008). 


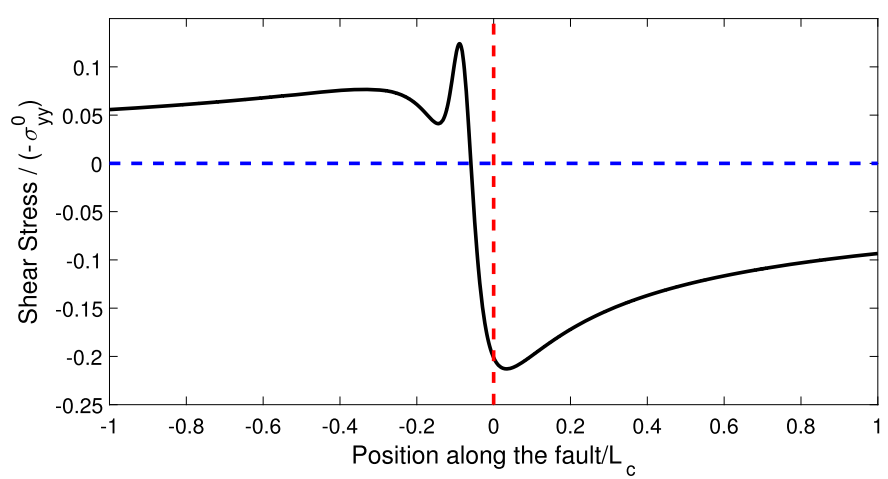

(a)

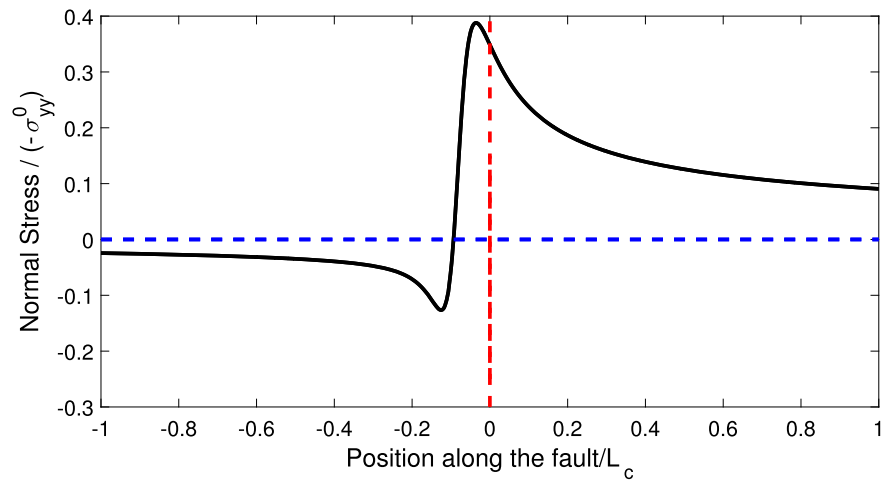

(b)

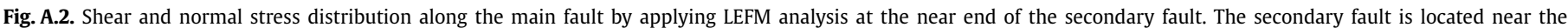

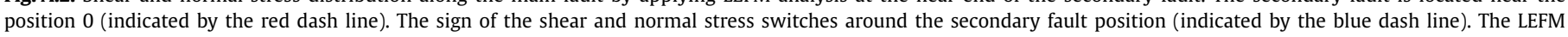
analysis gives a stress perturbation pattern that is in qualitative agreement with the results from numerical simulations.

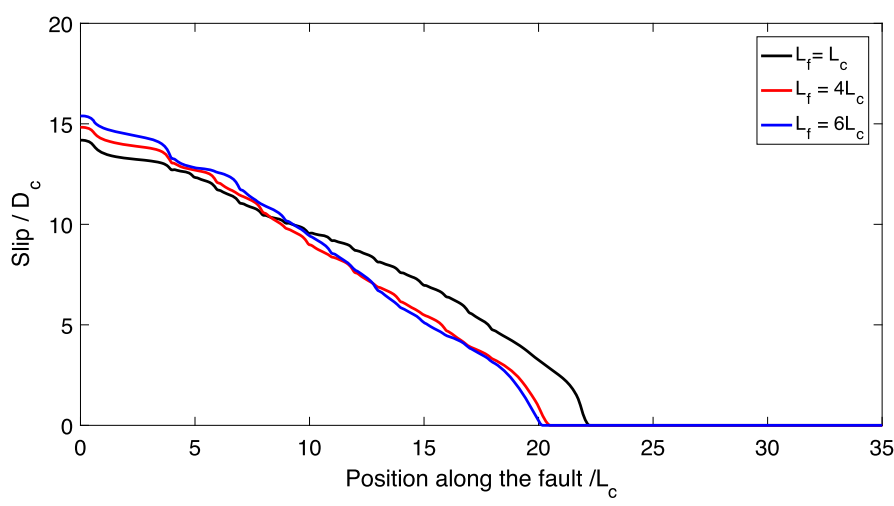

(a)

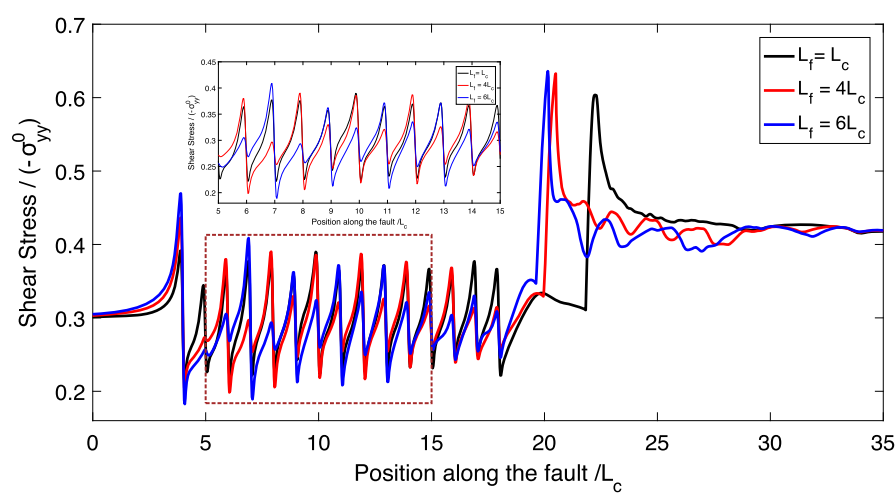

(c)

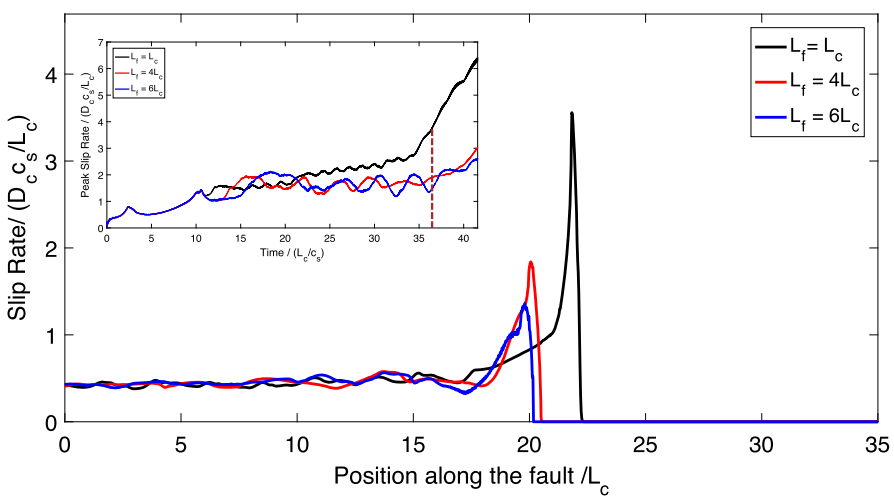

(b)

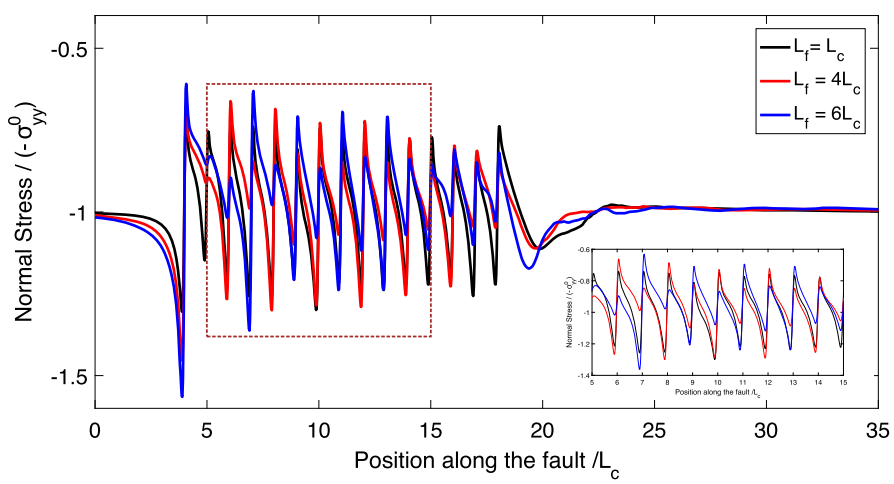

(d)

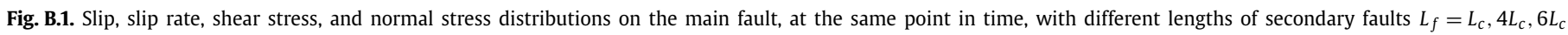

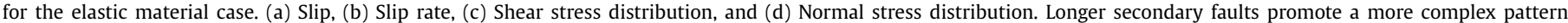
of stress perturbations on the main fault and lead to further reduction in the main rupture propagation speed. 


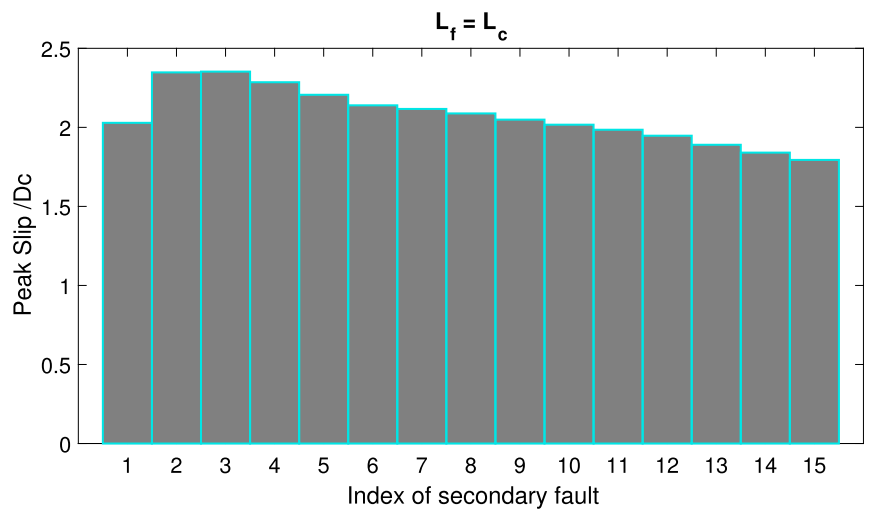

(a)

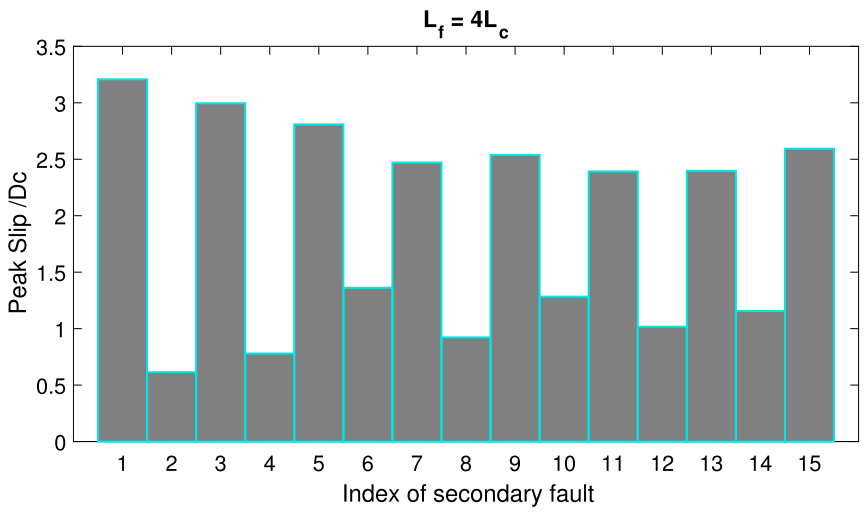

(b)

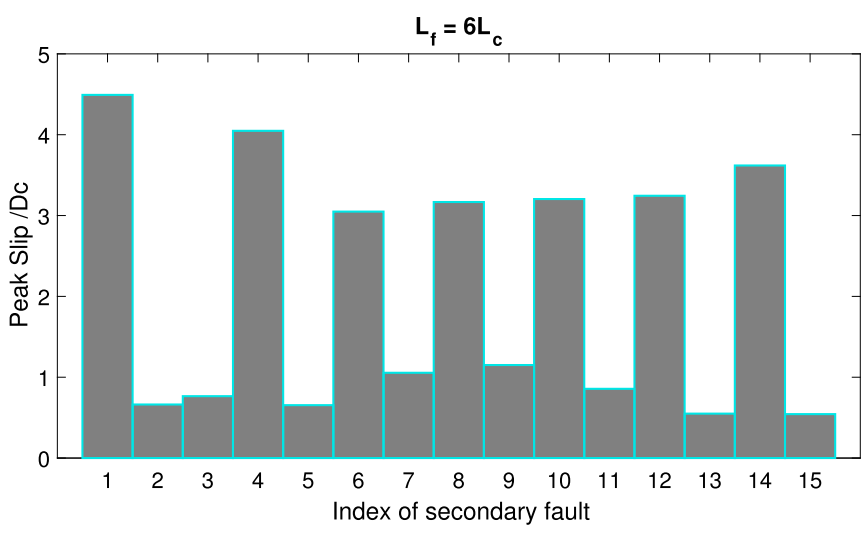

(c)

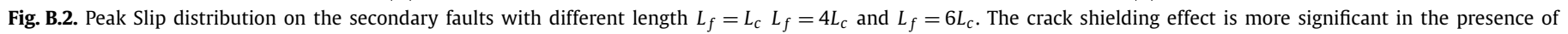
longer secondary faults.

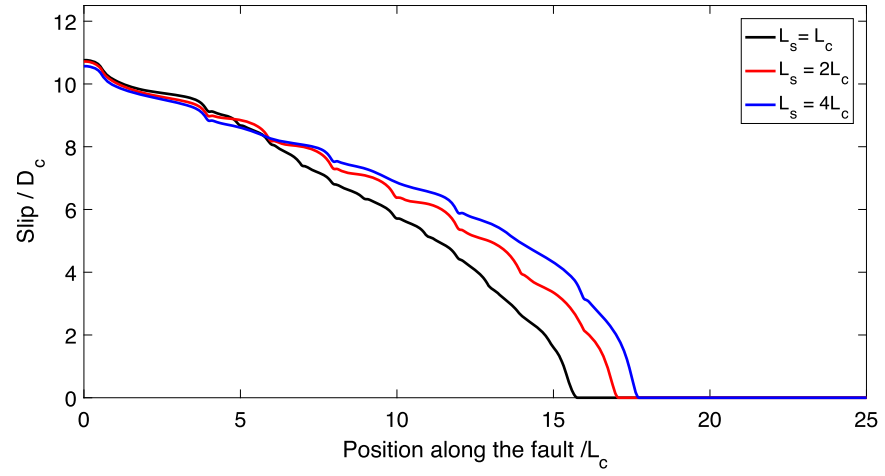

(a)

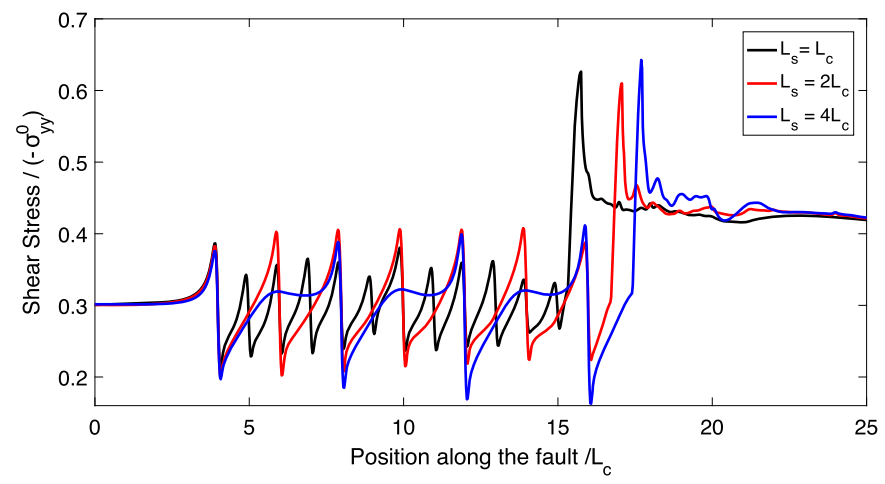

(c)

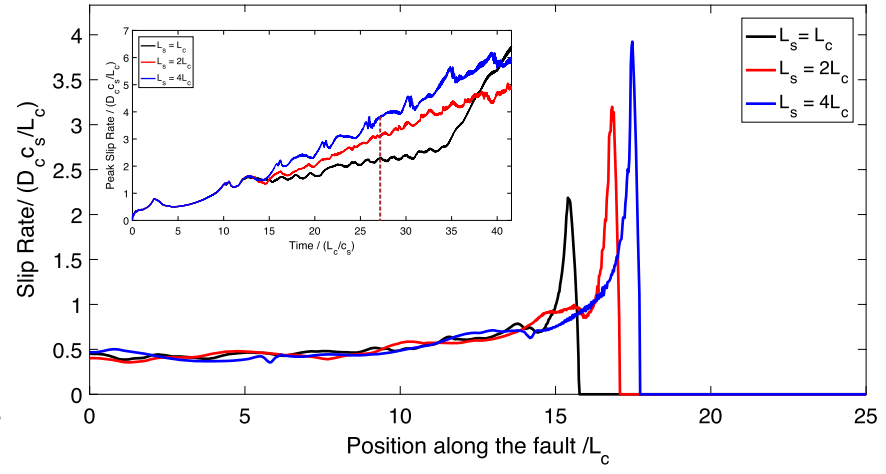

(b)

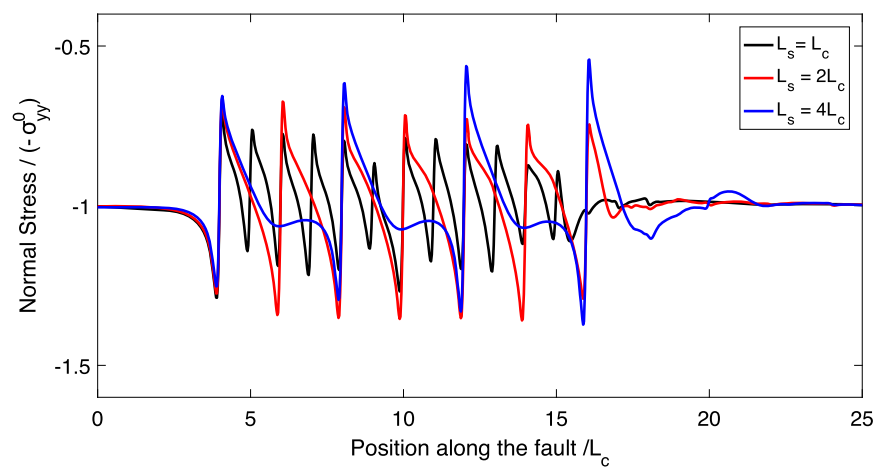

(d)

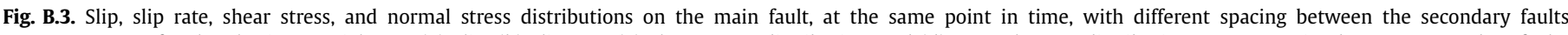

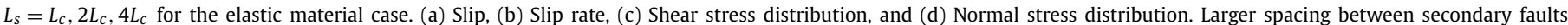
promote stronger perturbations in the stress and slip rate on the main fault. 


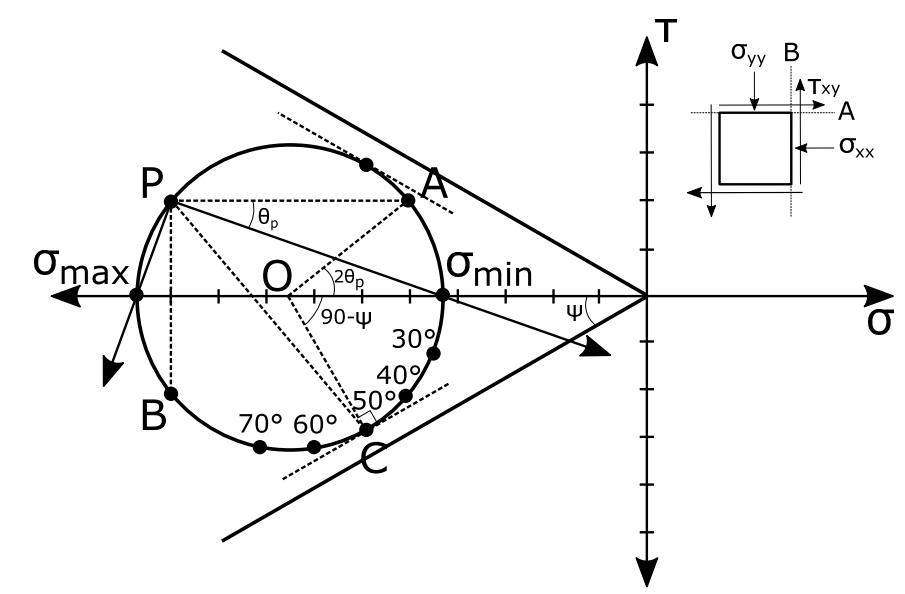

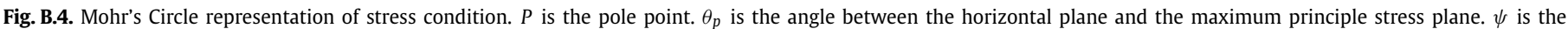
internal friction angle. Secondary faults with different angles are marked as black dots on the circle.

Table B.1

The resolved stress and strength parameter on the secondary fault for different angle. Note that negative sign in the normal stress indicates compression and negative sign in the shear stress indicates left lateral shear.

\begin{tabular}{llll}
\hline Secondary fault angle $\theta_{f}$ & Resolved Normal Stress & Resolved Shear Stress & Strength Parameter $S$ \\
\hline $30^{\circ}$ & $-45.18 \mathrm{MPa}$ & $-11.65 \mathrm{MPa}$ & -8.12 \\
$40^{\circ}$ & $-50.96 \mathrm{MPa}$ & $-21.15 \mathrm{MPa}$ & 1.61 \\
$50^{\circ}$ & $-59.65 \mathrm{MPa}$ & $-28.09 \mathrm{MPa}$ & 0.75 \\
$60^{\circ}$ & $-70.18 \mathrm{MPa}$ & $-31.65 \mathrm{MPa}$ & 0.99 \\
$70^{\circ}$ & $-81.30 \mathrm{MPa}$ & $-31.39 \mathrm{MPa}$ & 2.48 \\
\hline
\end{tabular}

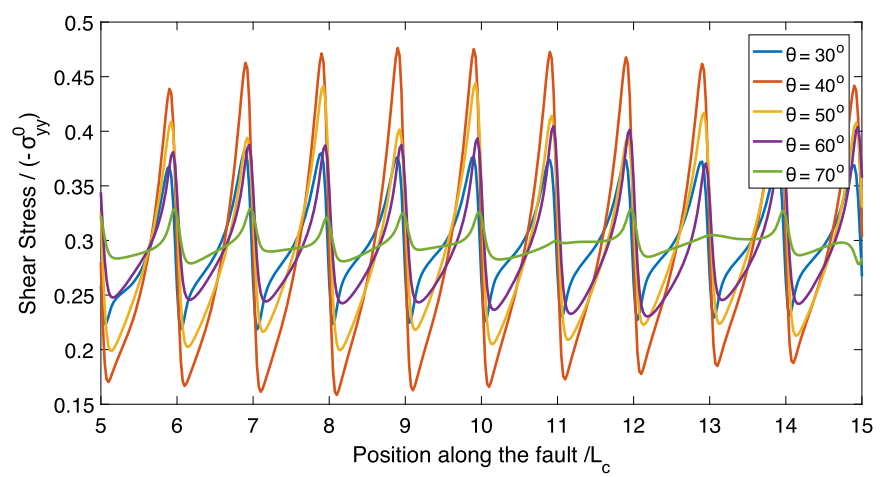

(a)

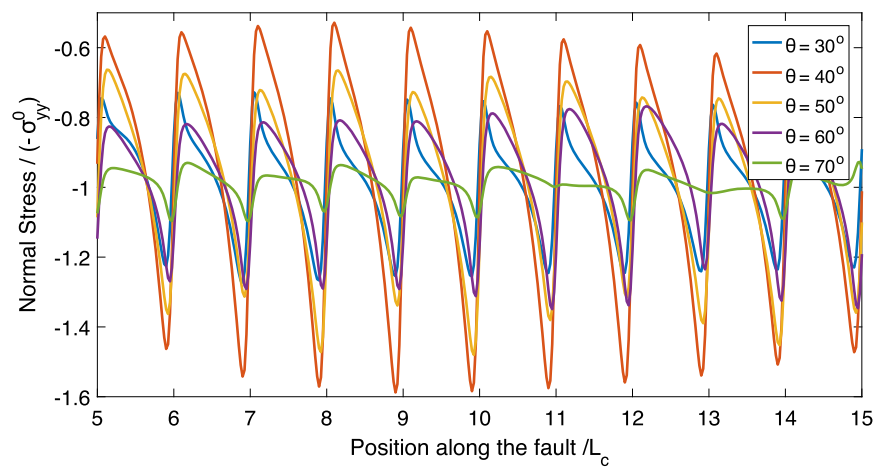

(b)

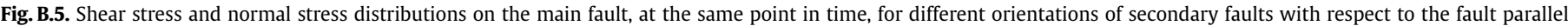

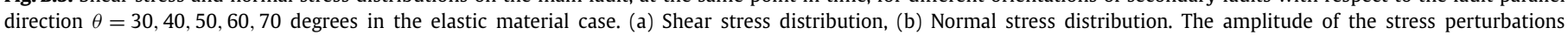
decrease as the secondary faults rotate away from the optimally oriented shear plane direction $(\theta=50$ degree).

\section{Effect of spacing distance between secondary faults}

Fig. B.3 shows a snapshot of slip, slip rate, shear stress, and normal stress distribution on the main fault at a given instant of time for three cases of secondary faults spacing $L_{s}=L_{c} ; 2 L_{c} ; 4 L_{c}$. As shown in Fig. B.3(c) and B.3(d), as the spacing between the sec- ondary faults increases, the amplitude of perturbations in the shear and normal stresses on main fault increases since each secondary fault accumulates more slip on average than in the case of smaller spacing. With smaller spacing between the secondary faults, the secondary faults are more effective in decelerating the rupture on 


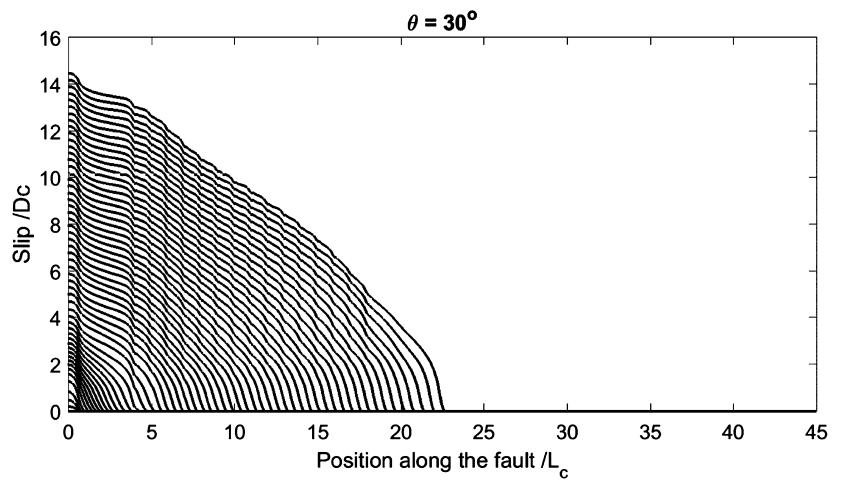

(a)

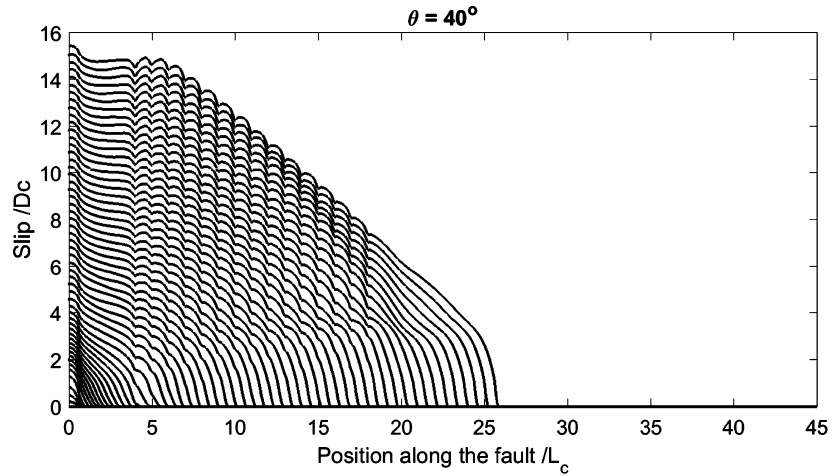

(b)

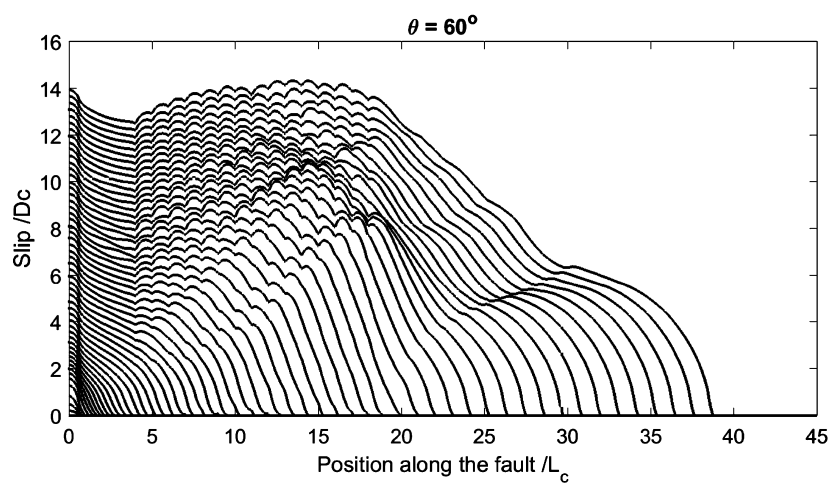

(d)

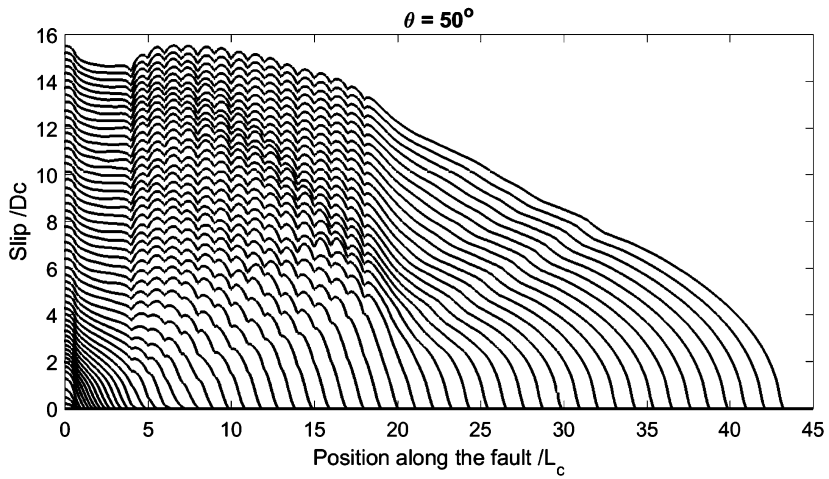

(c)

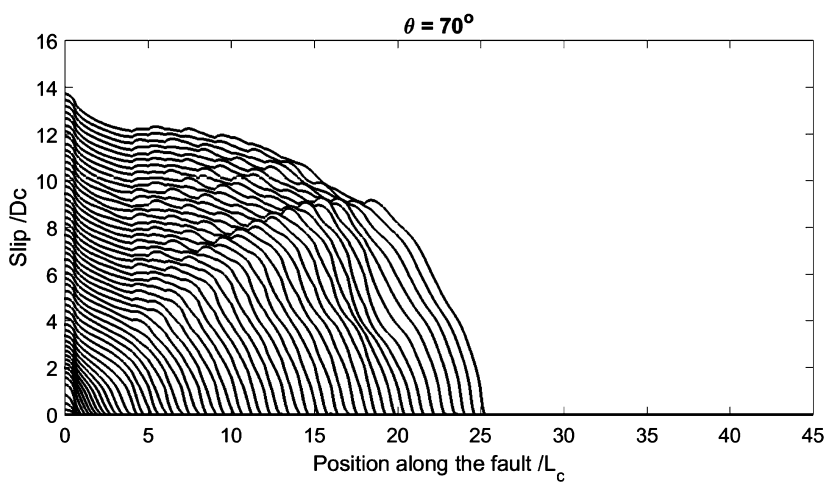

(e)

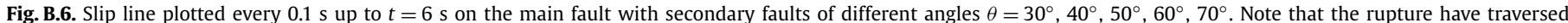

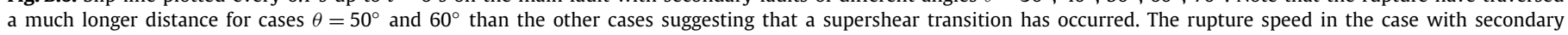
faults $\theta=50^{\circ}$ was found to be $0.92 c_{p}$.

the main fault. The insert in Fig. B.3(b) shows that with the increased spacing, the oscillations in the slip rate are spaced at a larger distance but their amplitude increases.

\section{Effect of secondary fault angle with respect to the main fault}

While the angle that a secondary fault makes with the main fault may be arbitrary, here we explore a number of different secondary faults orientation that vary around the direction of optimally oriented shear plane computed using the background tectonic stress field and a Mohr-Coulomb failure criterion.

$\theta=45^{\circ}+\theta_{p}-\frac{\psi}{2}$

In Eq. (B.1) above, $\psi$ is the angle of internal friction, and $\theta_{p}$ is the angle that the maximum principal stress makes with the horizontal direction. Fig. B.4 shows the Mohr's circle representing the state of the background stress in the domain. For the parameters shown in Table 1, and as demonstrated by the Mohr's circle, the direction of optimally oriented shear plane makes approximately a 50 degree angle clockwise with the direction of the main fault. Accordingly, we consider four cases of orientation of the branching faults, $\theta=40^{\circ}, 50^{\circ}, 60^{\circ}$, and $70^{\circ}$, in addition to the default case, $\theta=30^{\circ}$, discussed earlier. The resolved normal stress, shear stress and the strength parameter $S$ on the secondary faults are summarized in Table B.1. Fig. B.5 suggests that the amplitude of the shear and normal stress fluctuations on the main fault have a nonmonotonic trend as the secondary faults are rotated away from the main fault. The case of secondary faults with $\theta=40^{\circ}$ results in the largest amplitude of stress perturbations on the main fault among all the angles considered here. By investigating the slip evolution on the main fault (Fig. B.6), we have found that the main fault rupture has transitioned into supershear mode when 


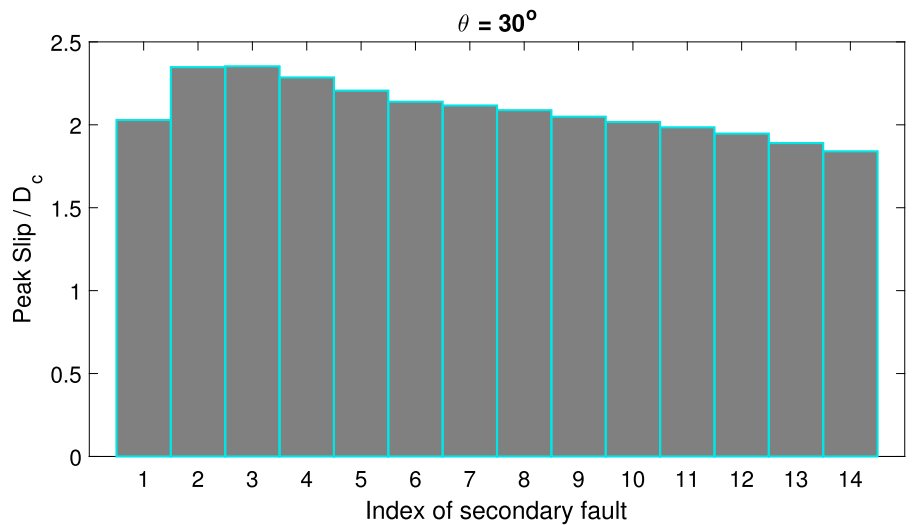

(a)

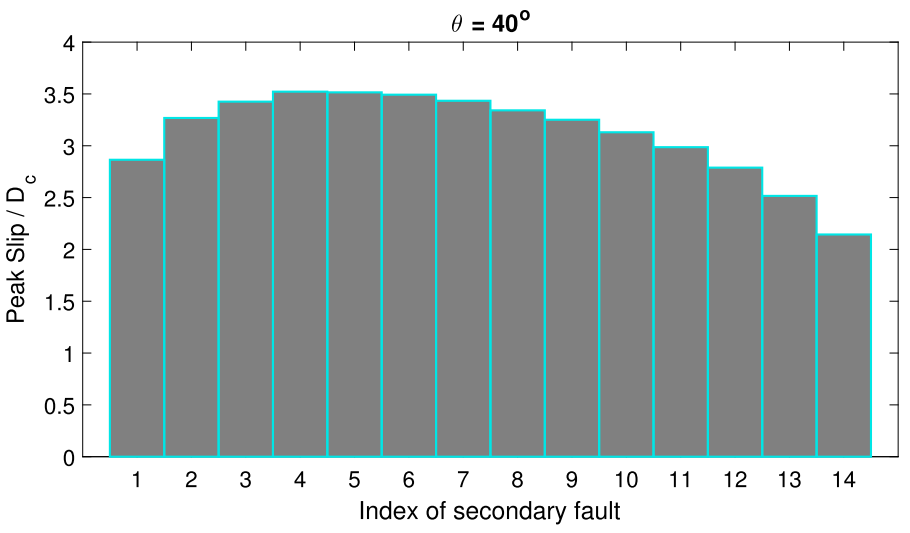

(b)

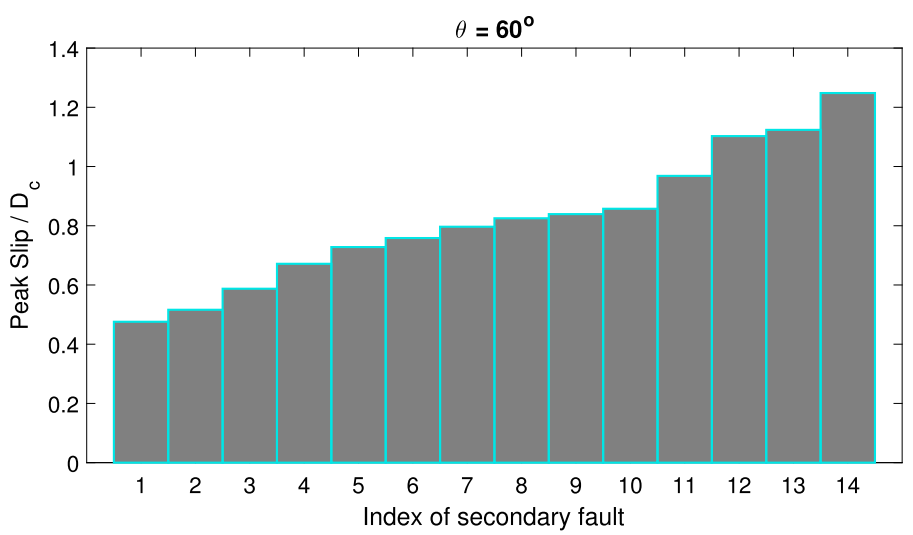

(d)

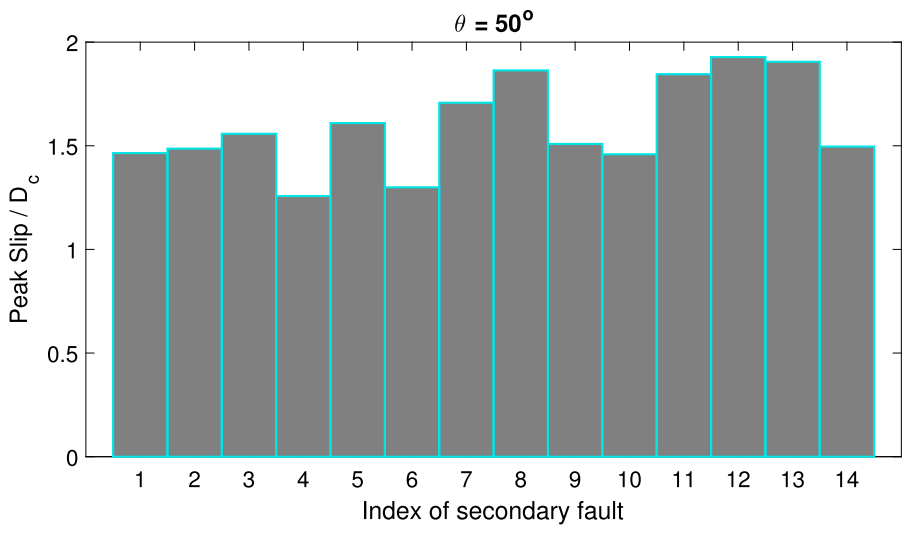

(c)

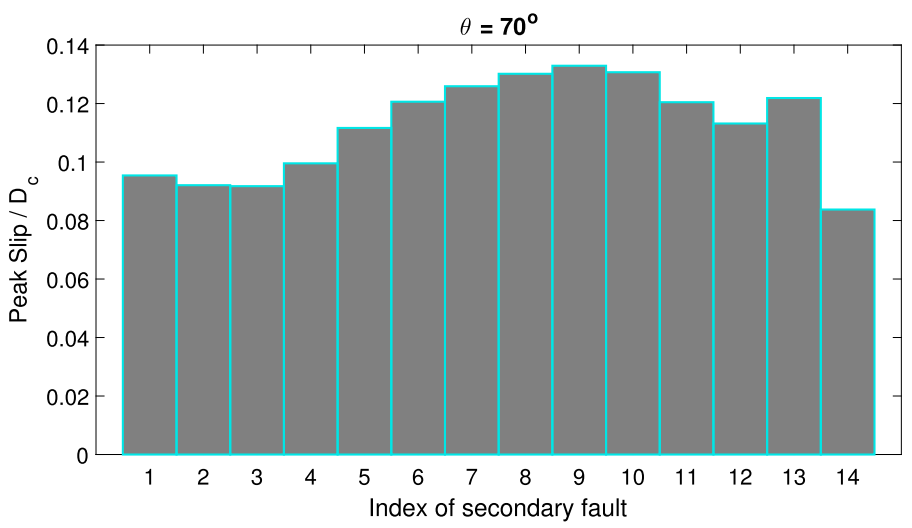

(e)

Fig. B.7. Peak Slip distribution on the secondary faults with different angles $\theta=30^{\circ}, 40^{\circ}, 50^{\circ}, 60^{\circ}, 70^{\circ}$.

the branch angles are $\theta=50^{\circ}$ and $\theta=60^{\circ}$ while it remains subshear in all the other cases $\left(\theta=30^{\circ}, 40\right.$, and 70). The resulting slip distribution across the secondary faults for the different branch angles are given by the histograms in Fig. B.7. The average slip on the secondary faults increases as the branch angle moves towards the optimal orientation (i.e. from $\theta=30^{\circ}$ to $\theta=40^{\circ}$ ) which is consistent with the increase in the amplitude of stress perturbations on the main fault. Surprisingly, however, the average slip on the secondary faults is lower at $\theta=50^{\circ}$ (the optimal orientation according to the background stress state) and $\theta=60^{\circ}$. The reduction in slip on the secondary faults in these cases, despite favorable orientation, is hypothesized to be due to the supershear transition on the main fault which leads to: (1) amplification of slip on the main fault, and (2) rapid exit of the main fault rupture tip from the fish bone region reducing the exposure time of the secondary faults to the impulsive dynamic loading from the main fault rupture tip. This is accompanied by a reduction in the amplitude of stress perturbations on the main fault compared to the case of $\theta=40^{\circ}$. Finally, as the branch angle further increases (e.g. $\theta=70^{\circ}$ ), the resolved shear stress starts to decrease while the resolved normal stress continues to increase. This makes it more difficult to trigger slip on the secondary branches. Indeed, the case for $\theta=70^{\circ}$ has much smaller average slip value (almost an order of magnitude less) than all the other cases. As a result, the stress 
perturbations on the main fault in this case is also the smallest. Understanding supershear transition mechanisms in fault systems with complex topology, like the one considered here, and implications for slip partitioning and stress transfer, will be the focus of a future investigation.

\section{Appendix C. Supplementary material}

Supplementary material related to this article can be found online at https://doi.org/10.1016/j.epsl.2019.07.005.

\section{References}

Ando, R., Yamashita, T., 2007. Effects of mesoscopic-scale fault structure on dynamic earthquake ruptures: dynamic formation of geometrical complexity of earthquake faults. J. Geophys. Res. 112 (B9), B09303.

Andrews, D.J., 1976. Rupture propagation with finite stress in antiplane strain. J. Geophys. Res. 81 (20), 3575.

Andrews, D.J., 2005. Rupture dynamics with energy loss outside the slip zone. J. Geophys. Res. 110 (B1), B01307.

Ben-Zion, Y., Sammis, C.G., 2003. Characterization of fault zones. Pure Appl. Geophys. 160 (3), 677-715.

Ben-Zion, Y., Shi, Z., 2005. Dynamic rupture on a material interface with spontaneous generation of plastic strain in the bulk. Earth Planet. Sci. Lett. 236 (1-2), 486-496.

Berenger, J.-P., 1994. A Perfectly Matched Layer for the Absorption of Electromagnetic Waves.

Bettess, P., 1977. Infinite elements. Int. J. Numer. Methods Eng. 11 (1), 53-64.

Bhat, H.S., Dmowska, R., Rice, J.R., Kame, N., 2004. Dynamic slip transfer from the Denali to totschunda faults, Alaska: testing theory for fault branching. Bull. Seismol. Soc. Am. 94 (6 SUPPL), B:202-213.

Bhat, H.S., Rosakis, A.J., Sammis, C.G., 2012. A micromechanics based constitutive model for brittle failure at high strain rates. J. Appl. Mech. 79 (3), 031016.

Biegel, R., Bhat, H., Sammis, C., Rosakis, A., 2010. The effect of asymmetric damage on dynamic shear rupture propagation I: no mismatch in bulk elasticity. Tectonophysics 493 (3-4), 254-262.

Biegel, R.L., Sammis, C.G., Rosakis, A.J., 2007. Interaction of a dynamic rupture on a fault plane with short frictionless fault branches. Pure Appl. Geophys. 164 (10), 1881-1904.

Bielak, J., Loukakis, K., Hisada, Y., Yoshimura, C., 2003. Domain reduction method for three-dimensional earthquake modeling in localized regions, Part I: theory. Bull. Seismol. Soc. Am. 93 (2), 817-824.

Brodsky, E.E., Kirkpatrick, J.D., Candela, T., 2016. Constraints from fault roughness on the scale-dependent strength of rocks. Geology 44 (1), 19-22.

Chen, X., 1995. Near-Field Ground Motion From the Landers Earthquake.

Chester, F.M., Evans, J.P., Biegel, R.L., 1993. Internal structure and weakening mechanisms of the San Andreas Fault. J. Geophys. Res., Solid Earth 98 (B1), 771-786.

Das, S., Aki, K., 1977. A numerical study of two-dimensional spontaneous rupture propagation. Geophys. J. Int. 50 (3), 643-668.

Day, S.M., 1982. Three-dimensional finite difference simulation of fault dynamics: rectangular faults with fixed rupture velocity. Bull. Seismol. Soc. Am. 72 (3), 705-727.

DeDontney, N., Rice, J.R., Dmowska, R., 2012. Finite element modeling of branched ruptures including off-fault plasticity. Bull. Seismol. Soc. Am. 102 (2), 541-562.

Dieterich, J.H., 1979. Modeling of rock friction: 1. Experimental results and constitutive equations. J. Geophys. Res., Solid Earth 84 (B5), 2161-2168.

Drucker, D.C., Prager, W., 1952. Soil mechanics and plastic analysis or limit design. Q. Appl. Math. 10 (2), 157-165.

Dunham, E.M., Belanger, D., Cong, L., Kozdon, J.E., 2011a. Earthquake ruptures with strongly rate-weakening friction and off-fault plasticity, Part 1: planar faults. Bull. Seismol. Soc. Am. 101 (5), 2296-2307.

Dunham, E.M., Belanger, D., Cong, L., Kozdon, J.E., 2011b. Earthquake ruptures with strongly rate-weakening friction and off-fault plasticity, Part 2: nonplanar faults. Bull. Seismol. Soc. Am. 101 (5), 2308-2322.

Duru, K., Dunham, E.M., 2016. Dynamic earthquake rupture simulations on nonplanar faults embedded in 3D geometrically complex, heterogeneous elastic solids. J. Comput. Phys. 305 (C), 185-207.

Geubelle, P.H., Rice, J.R., 1995. A spectral method for three-dimensional fracture problems. J. Mech. Phys. Solids 43 (11), 1791-1824.

Hajarolasvadi, S., Elbanna, A.E., 2017. A new hybrid numerical scheme for modelling elastodynamics in unbounded media with near-source heterogeneities. Geophys. J. Int. 211 (2), 851-864.

Hok, S., Campillo, M., Cotton, F., Favreau, P., Ionescu, I., 2010. Off-fault plasticity favors the arrest of dynamic ruptures on strength heterogeneity: two-dimensional cases. Geophys. Res. Lett. 37 (2), n/a-n/a.

Ida, Y., 1972. Cohesive force across the tip of a longitudinal-shear crack and Griffith's specific surface energy. J. Geophys. Res. 77 (20), 3796-3805.
Kame, N., Rice, J.R., Dmowska, R., 2003. Effects of prestress state and rupture velocity on dynamic fault branching. J. Geophys. Res., Solid Earth 108 (B5), 1-21.

Klinger, Y., Okubo, K., Vallage, A., Champenois, J., Delorme, A., Rougier, E., Lei, Z. Knight, E.E., Munjiza, A., Satriano, C., Baize, S., Langridge, R., Bhat, H.S., 2018. Earthquake damage patterns resolve complex rupture processes. Geophys. Res. Lett., 279-287.

Liu, F., Borja, R.I., 2009. An extended finite element framework for slow-rate frictional faulting with bulk plasticity and variable friction. Int. J. Numer. Anal. Methods Geomech. 33 (13), 1535-1560.

Liu, F., Borja, R.I., 2013. Extended finite element framework for fault rupture dynamics including bulk plasticity. Int. J. Numer. Anal. Methods Geomech. 37 (18), 3087-3111.

Liu, Y., Lapusta, N., 2008. Transition of mode II cracks from sub-Rayleigh to intersonic speeds in the presence of favorable heterogeneity. J. Mech. Phys. Solids 56 (1), 25-50.

Lysmer, J., Kuhlemeyer, R.L., 1969. Finite dynamic model for infinite media. J. Eng Mech. Div. 95, 859-878

Ma, X., Elbanna, A., 2018. Strain localization in dry sheared granular materials: a compactivity-based approach. Phys. Rev. E 98 (2), 22906.

Ma, X., Hajarolasvadi, S., Albertini, G., Kammer, D.S., Elbanna, A.E., 2018. A hybrid finite element-spectral boundary integral approach: applications to dynamic rupture modeling in unbounded domains. Int. J. Numer. Anal. Methods Geomech. (August), 1-22.

Ngo, D., Huang, Y., Rosakis, A., Griffith, W.A., Pollard, D., 2012. Off-fault tensile cracks: a link between geological fault observations, lab experiments, and dynamic rupture models. J. Geophys. Res., Solid Earth 117 (B1), n/a-n/a.

Pelties, C., de la Puente, J., Ampuero, J.-P., Brietzke, G.B., Käser, M., 2012. Threedimensional dynamic rupture simulation with a high-order discontinuous Galerkin method on unstructured tetrahedral meshes. J. Geophys. Res., Solid Earth 117 (B2), n/a-n/a.

Pelties, C., Gabriel, A.-A., Ampuero, J.-P., 2014. Verification of an ADER-DG method for complex dynamic rupture problems. Geosci. Model Dev. 7 (3), 847-866.

Poliakov, A.N.B., Dmowska, R., Rice, J.R., 2002. Dynamic shear rupture interactions with fault bends and off-axis secondary faulting. J. Geophys. Res. 107 (B11), ESE 6-1-ESE 6-18.

Preuss, S., Herrendörfer, R., Gerya, T., Ampuero, J.-P., Dinther, Y.v., 2019. Seismic and aseismic fault growth lead to different fault orientations. EarthArXiv.

Rempe, M., Mitchell, T., Renner, J., Nippress, S., Ben-Zion, Y., Rockwell, T., 2013. Damage and seismic velocity structure of pulverized rocks near the San Andreas Fault. J. Geophys. Res., Solid Earth 118 (6), 2813-2831.

Rousseau, C.-E., Rosakis, A.J., 2009. Dynamic path selection along branched faults: experiments involving sub-Rayleigh and supershear ruptures. J. Geophys. Res. 114 (B8), B08303.

Rowe, C.D., Ross, C., Swanson, M.T., Pollock, S., Backeberg, N.R., Barshi, N.A., Bate, C.E., Carruthers, S., Coulson, S., Dascher-Cousineau, K., Harrichhausen, N., Peña Castro, A.F., Nisbet, H., Rakoczy, P., Scibek, J., Smith, H., Tarling, M.S., Timofeev, A., Young, E., 2018. Geometric complexity of earthquake rupture surfaces preserved in pseudotachylyte networks. J. Geophys. Res., Solid Earth 123 (9), 7998-8015.

Rudnicki, J.W., Rice, J.R., 1975. Conditions for the localization of deformation in pressure-sensitive dilatant materials. J. Mech. Phys. Solids 23 (6), 371-394.

Ruina, A., 1983. Slip instability and state variable friction laws. J. Geophys. Res., Solid Earth 88 (B12), 10359-10370.

Savage, H.M., Brodsky, E.E., 2011. Collateral damage: evolution with displacement of fracture distribution and secondary fault strands in fault damage zones. J. Geophys. Res., Solid Earth 116 (3).

Shi, Z., Day, S.M., 2013. Rupture dynamics and ground motion from 3-D rough-fault simulations. J. Geophys. Res., Solid Earth 118 (3), 1122-1141.

Sowers, J.M., Unruh, J.R., Lettis, W.R., Rubin, T.D., 1911. Relationship of the kickapoo fault to the Johnson Valley and Homestead Valley faults, San Bernardino County, California. Bull. Seismol. Soc. Am. 84 (3), 528-536.

Sun, C.-T., Jin, Z.-H., 2012. Fracture Mechanics. Academic Press.

Suzuki, T., 2013. Damage-tensor-based nondimensional parameters governing secondary faulting behavior. Tectonophysics 600, 205-216.

Tal, Y., Hager, B.H., Ampuero, J.P., 2018. The effects of fault roughness on the earthquake nucleation process. J. Geophys. Res., Solid Earth 123 (1), 437-456.

Templeton, E.L., Rice, J.R., 2008. Off-fault plasticity and earthquake rupture dynamics: 1. Dry materials or neglect of fluid pressure changes. J. Geophys. Res., Solid Earth 113 (9), 1-19.

Uenishi, K., Rice, J.R., 2003. Universal nucleation length for slip-weakening rupture instability under nonuniform fault loading. J. Geophys. Res., Solid Earth 108 (B1).

Uphoff, C., Rettenberger, S., Bader, M., Madden, E.H., Ulrich, T., Wollherr, S., Gabriel, A.-A., 2017. Extreme scale multi-physics simulations of the tsunamigenic 2004 Sumatra megathrust earthquake. In: Proceedings of the International Conference for High Performance Computing, Networking, Storage and Analysis on - SC '17. ACM Press, New York, New York, USA, pp. 1-16.

Wald, D.J., Heaton, T.H., 1994. Spatial and temporal distribution of slip for the 1992 Landers, California, earthquake. Bull. Seismol. Soc. Am. 84 (3), 668-691. 
Xu, S., Ben-Zion, Y., Ampuero, J.P., Lyakhovsky, V., 2015a. Dynamic ruptures on a frictional interface with off-fault brittle damage: feedback mechanisms and effects on slip and near-fault motion. Pure Appl. Geophys. 172 (5), 1243-1267.

Xu, S., Fukuyama, E., Ben-Zion, Y., Ampuero, J.-P., 2015b. Dynamic rupture activation of backthrust fault branching. Tectonophysics 644 (645), 161-183.
Yoshimura, C., Bielak, J., Hisada, Y., Fernández, A., 2003. Domain reduction method for three-dimensional earthquake modeling in localized regions, Part II: verification and applications. Bull. Seismol. Soc. Am. 93 (2), 825-841. 